\title{
Anderson localization in anisotropic random media
}

\author{
Zhao-Qing Zhang* and Qian-Jing Chu \\ Institute of Physics, Academia Sinica, Beijing, China \\ and Center of Theortical Physics, CCAST (World Laboratory), P.O. Box 8730, Beijing, China
}

Weige Xue and Ping Sheng

Exxon Research and Engineering Company, Route 22 East, Clinton Township, Annandale, New Jersey 08801

(Received 13 March 1990)

\begin{abstract}
Anderson localization in systems where the randomness is anisotropic and intermediate between the different dimensions is studied both analytically and numerically. As a function of the anisotropy parameter $\theta$, which interpolates between a one-dimensional randomly layered system and threedimensional isotropic randomness, our results indicate the existence of a Fermi-energy-dependent critical $\theta_{c}$ below which the wave function is localized for arbitrarily small randomness. An interesting reentry phenomenon is found in the localization phase diagram near the band edge, where the density of states is small. Expressions for the localization lengths are obtained analytically in the localized regime when the randomness is small. The behavior of the localization length in the layering direction is found to follow the simple one-dimensional result, while in the lateral direction the localization length behaves differently from the standard two-dimensional result. Physical arguments are presented to make plausible the above behaviors. Our model is contrasted with the anisotropic hopping model. Significant differences are noted.
\end{abstract}

\section{INTRODUCTION}

In the past decade tremendous progress has been made in understanding the phenomenon of Anderson localization for noninteracting electrons in random potentials. ${ }^{1}$ The single-parameter scaling theory proposed by Abrahams et al. ${ }^{2}$ has been confirmed by both analytic ${ }^{3}$ and numerical approaches. ${ }^{4-6}$ It is now believed that all states are localized in dimensions one and two for any amount of randomness.

The model generally adopted for the study of localization is the tight-binding Anderson Hamiltonian ${ }^{7}$

$$
H=\sum_{\alpha} \varepsilon_{\alpha} a_{\alpha}^{\dagger} a_{\alpha}+t \sum_{\mathrm{NN}} a_{\alpha}^{\dagger} a_{\beta},
$$

where $a_{\alpha}^{\dagger}, a_{\alpha}$ are the creation and annihilation operators, respectively, $\alpha, \beta$ are indices for sites on an $n$-dimensional lattice, and NN denotes nearest neighbors. The hopping matrix $t$ is usually assumed to be constant, and the potential energy $\varepsilon_{\alpha}$ at site $\alpha$ is random with a distribution $P\left(\varepsilon_{\alpha}\right)$, which is either flat or Gaussian. For the above model, much has been learned about the localization behavior and its dimensional dependence. ${ }^{8}$ In this work, we propose a new model, where the randomness of site energy is anisotropic and intermediate between different dimensions, e.g., a random superlattice with lateral inhomogeneities. ${ }^{9}$ In a three-dimensional (3D) simple cubic lattice, the site energy $\varepsilon_{\alpha}$ is written as the sum of two additive components:

$$
\varepsilon_{\alpha}=\theta \eta_{i z}+(1-\theta) \gamma_{z}
$$

where $z$ denotes the layering axis, and $i$ denotes the transverse direction $x$ and $y . \quad \gamma$ and $\eta$ are random variables with the same flat distribution $P$ of width $W$, i.e.,

$$
P\left(\gamma_{z}\right)=P\left(\eta_{i z}\right)=P(x)=\left\{\begin{array}{l}
1 / W,|x| \leq W / 2 \\
0, \text { otherwise, }
\end{array}\right.
$$

$\theta$ is an anisotropy parameter, varying between 0 and 1 , which interpolates the randomness between a $1 \mathrm{D}$ layered $(\theta=0)$ and a $3 \mathrm{D}$ isotropic $(\theta=1)$ system. Physically, our model corresponds to predominately layered systems, such as the Earth's subsurface or random superlattices, with lateral inhomogeneities that may be inherent to the system or deliberately introduced.

The above model is studied both analytically and numerically in this work. In the analytical approach, the self-consistent theory of Vollhardt and Wolfle ${ }^{10}$ (VW) is used in conjunction with the self-consistent Born approximation (SCBA). By taking into account the coherentbackscattering effect, the theory of $\mathrm{VW}$ has been shown to be very successful in determining the localization phase diagram of isotropic random models. ${ }^{11}$ This diagrammatical theory has also been used to study the localization properties of phonons ${ }^{12}$ and various kinds of classical waves. ${ }^{13}$ For the model proposed here, the site energy $\varepsilon_{\alpha}$ of Eq. (2) contains a constant part, i.e., $(1-\theta) \gamma_{z}$, in every layer. Since the infinite-range correlation of this term inhibits the use of coherent-potential approximation (CPA) in obtaining the averaged singleparticle Green's function, SCBA is used instead. SCBA is known to be valid when the randomness is small and has been used previously in the study of classical waves ${ }^{14}$ and phonons ${ }^{15}$ with long-range correlations. Thus we expect our analytical results to be at least qualitatively correct. In the numerical approach, the localization phase diagrams are determined by using the finite-size scaling method, which has become standard in the study of localization phase diagrams in disordered systems. ${ }^{5,6}$ 
Our results show that anisotropy yields novel characteristics that may have potential applications. In particular, a Fermi-energy-dependent critical $\theta_{c}$ is found, below which the wave function is localized for arbitrarily small randomness, and the system behaves basically as a 1D layered material. Above $\theta_{c}$ the system is shown to acquire the $3 \mathrm{D}$ characteristics of exhibiting mobility edges. In the region below $\theta_{c}$, an interesting reentry phenomenon exists near the band edge, where the density of states (DOS) is small.

Our anisotropic model is shown to be not describable by a single-parameter scaling theory. The predicted behaviors are also noted to be significantly different from the previously studied anisotropic models, ${ }^{16-20}$ where the hopping matrix elements are different in the layering and lateral directions but the site energy is isotropically random. In these models it has been shown that the problem can be mapped to a single-parameter scaling theory through an anisotropic scale transformation. Therefore, the system exhibits 3D Anderson transition as soon as the hopping deviates from strict one (or two) dimensionality, ${ }^{20}$ i.e., at $\theta_{c}=0$.

When the randomness is small, asymptotic behavior of the localization lengths in the region below $\theta_{c}$ is obtained. As a function of randomness $W$, the localization length in the layering direction behaves like a 1D system, while in the lateral direction the behavior differs from the standard 2D result. For a fixed $W$, the localization length in the layering direction can be made to diverge with a power-law exponent $\frac{1}{2}$ by increasing the value of lateral inhomogeneities.

In Sec. II, the formulation for the conductivity, mobility edge, and localization lengths are given in the framework of VW theory. A description of the numerical method is given in Sec. III. Section IV contains the results and the related discussions. Conclusions are given in Sec. $\mathrm{V}$.

\section{FORMULATION}

Before delving into the details of the theory, we first outline the overall road map. For the anisotropic diagonally disordered system described by Eqs. (1)-(3), the averaged single-particle scattering (in the context of SCBA) contains two parts: the $3 \mathrm{D}$ isotropic scattering and the 1D scattering in the layering direction with strengths $W^{2} \theta^{2} / 12$ and $W^{2}(1-\theta)^{2} / 12$, respectively. The Kubo formula ${ }^{21}$ is used to evaluate the conductivity tensor. The expressions for the bare conductivities in both the layering and lateral directions are obtained by summing the ladder diagrams. The coherent backscattering effect for localization is then taken into account by including the maximally crossed diagrams (MCD's) in the particle-hole channel as is usually done. However, there is an important difference from the isotropic case in that there is a class of MCD that contains only the 1D scattering. These diagrams reduce the conductivity in the layering direction but have no effect on the lateral direction, since they conserve the lateral momentum. It is this important difference that gives all the novel characteristics of the model mentioned in the Introduction. Following
VW, the bare diffusion constants in the diffusion poles of the coherent-backscattering terms are renormalized to obtain a set of coupled self-consistent equations for conductivities (or diffusion constants) in both the layering and lateral directions. By setting to zero the renormalized conductivities, the equations to determine the mobility edges and localization lengths are then obtained.

\section{A. Single-particle Green's function}

The Hamiltonian of Eq. (1) can be rewritten in the momentum representation as

$$
H=H_{0}+H_{1}=\sum_{\mathbf{p}} \varepsilon(\mathbf{p}) a_{p}^{\dagger} a_{\mathrm{p}}+\sum_{\mathrm{p} \cdot \mathbf{q}} v(\mathbf{q}) a_{\mathrm{p}+\mathrm{q}}^{\dagger} a_{\mathrm{p}},
$$

with

$$
\varepsilon(\mathbf{p})=\sum_{\delta} \exp (-i \mathbf{p} \cdot \delta),
$$

and

$$
v(\mathbf{q})=\frac{1}{N} \Sigma_{\alpha} \varepsilon_{\alpha} \exp \left(-1 \mathbf{q} \cdot \mathbf{R}_{\alpha}\right),
$$

where $\delta$ denotes the vector pointing from a site to one of its nearest neighbors. $\mathbf{R}_{\alpha}$ is the spatial coordinate of site $\alpha$, and $N$ is the total number of sites in the lattice. In SCBA, the averaged single-particle Green's function (GF) and its self-energy $\Sigma$ can be derived from Eqs. (2)-(6):

$$
\begin{aligned}
R_{\mathbf{p}}^{ \pm}= & \left\langle G_{\mathbf{p}}^{ \pm}\right\rangle_{c}=\left\langle\left\langle\mathbf{p}\left|\frac{1}{E \pm i \eta-H}\right| \mathbf{p}\right\rangle\right\rangle_{c} \\
& =\frac{1}{E-\varepsilon(\mathbf{p})-\Sigma_{\mathbf{p}}^{ \pm}(E)}, \\
\Sigma_{p}^{ \pm}= & \sum_{\mathbf{q}}\langle v(\mathbf{q}) v(-\mathbf{q})\rangle_{c} R_{\mathbf{p}+\mathbf{q}}^{ \pm} \\
= & \frac{1}{N} \sum_{\mathbf{q}} S(\mathbf{q}) R_{\mathbf{p}+\mathbf{q}}^{ \pm} \\
= & \frac{W^{2} \theta^{2}}{12} \frac{1}{N} \sum_{\mathbf{p}^{\prime}} \frac{1}{E-\varepsilon\left(\mathbf{p}^{\prime}\right)-\Sigma_{\mathbf{p}_{\|}^{\prime}}^{ \pm}} \\
& +\frac{W^{2}(1-\theta)^{2}}{12} \frac{1}{N_{\perp}} \sum_{\mathbf{p}_{1}} \frac{1}{E-\varepsilon\left(\mathbf{p}_{\|}+\mathbf{p}_{\perp}\right)-\Sigma_{\mathbf{p}_{\|}}^{ \pm}},
\end{aligned}
$$

with

$$
S(\mathbf{q})=\frac{W^{2}}{12}\left[\theta^{2}+(1-\theta)^{2} N_{\|} \delta_{\mathbf{q}_{\|}, 0}\right] .
$$

Here $G^{+}$and $G^{-}$are, respectively, the retarded and advanced GF's. $E$ denotes the electron energy, $N_{\perp}$ is the number of layers, " $\perp$ " and " $\|$ " denote components of vector in the layering and lateral directions, respectively, and \langle\rangle$_{c}$ denotes configurational averaging. The derivation of Eq. (8) is given in Appendix A. In the presence of anisotropy $(\theta<1)$, the scattering strength function $S(\mathbf{q})$ is seen to contain two parts: one from the $3 \mathrm{D}$ isotropic scattering, with strength $W^{2} \theta^{2} / 12$ and the other from 1D scattering in the layering direction, which conserves the transverse momentum in the lateral direction and has strength $W^{2}(1-\theta)^{2} / 12$. Thus the self-consistent solution 
of Eq. (8) leads to a self-energy that depends not only on the energy but also on the transverse momentum $\mathbf{p}_{\|}$.

\section{B. Conductivities}

Knowing the averaged single-particle GF, we can now evaluate the conductivity by using the Kubo formula, ${ }^{21}$

$$
\begin{aligned}
\sigma_{j j}(\omega, E)=\frac{2 \pi e^{2}}{N} \int & d E\left[-\frac{\partial f}{\partial E}\right) \\
\times & \operatorname{Tr}\left\langle v_{j} \delta\left(E_{+}-H\right) v_{j} \delta\left(E_{-}-H\right)\right\rangle_{c},
\end{aligned}
$$

where $E_{ \pm}=E \pm \omega / 2, f$ is the Fermi distribution function, and $v_{j}$ is the velocity operator in the $j$ direction with the matrix element

$$
\left\langle\mathbf{p}^{\prime}\left|v_{j}\right| \mathbf{p}\right\rangle=\delta_{\mathbf{p}, \mathbf{p}^{\prime}} \boldsymbol{\nabla}_{p_{j}} \varepsilon(\mathbf{p})=\delta_{\mathbf{p}, \mathbf{p}} v_{j}(\mathbf{p})
$$

In this work we have set the Plank constant $h$ and lattice constant to be unity. Due to the anisotropy there are two different conductivities $\sigma_{\perp}\left(=\sigma_{z z}\right)$ and $\sigma_{\|}\left(=\sigma_{i i}\right)$ in the layering and transverse directions, respectively. By using the relation

$$
\delta\left(E_{ \pm}-H\right)=(i / 2 \pi)\left[G^{+}\left(E_{ \pm}\right)-G^{-}\left(E_{ \pm}\right)\right],
$$

the zero-temperature conductivity of Eq. (10) can be written as

$$
\sigma_{j j}(\omega, E) \cong \frac{2 \pi e^{2}}{N}\left(\phi_{j j}^{R A}+\phi_{j j}^{A R}\right),
$$

with

$$
\phi_{j j(\omega, E)}^{R A(A R)}=\frac{1}{4 \pi^{2}} \sum_{\mathbf{p}, \mathbf{p}^{\prime}} v_{j}(\mathbf{p})\left\langle\left\langle\mathbf{p}\left|G^{+(-)}\left(E_{+}\right)\right| \mathbf{p}^{\prime}\right\rangle_{v_{j}}\left(\mathbf{p}^{\prime}\right)\left\langle\mathbf{p}^{\prime}\left|G^{-1+)}\left(E_{-}\right)\right| \mathbf{p}\right\rangle\right\rangle_{c}
$$

where $E$ becomes the Fermi energy. We have neglected the terms like $\phi_{j j}^{R R}$ and $\phi_{j j}^{A A}$ because they are inherently small (since they have two poles on the same side). The ensuing calculation of $\phi_{j j}^{R A}$ and $\phi_{j j}^{A A}$ consists of evaluating two classes of diagrams: the ladder diagrams for the bare conductivities and the MCD's for the coherentbackscattering effect that gives rise to localization.

The ladder diagrams are shown in Fig. 1, where the solid lines are the averaged GF's. Each vertex, denoted by heavy solid line, contains two types of scattering: 3D and 1D scattering, which are denoted by dashed and dotted lines, respectively. For $\sigma_{1}$, only the diagram Fig. 1(a) contributes because all the other diagrams vanish after the summation of $\mathbf{p}$ and $\mathbf{p}^{\prime}$. In the static limit $(\omega \rightarrow 0)$ this diagram gives the following result for the bare conductivity $\sigma_{\perp}^{0}$ :

$$
\begin{aligned}
\sigma_{\perp}^{(0)}(E) & =\frac{e^{2}}{\pi} \frac{1}{N} \sum_{\mathbf{p}} v_{\perp}^{2}(\mathbf{p}) R_{\mathbf{p}}^{+}(E) R_{\mathbf{p}}^{-}(E) \\
& =\frac{e^{2}}{\pi} \frac{1}{N} \sum_{\mathbf{p}} v_{z}^{2}(\mathbf{p}) \frac{\Delta R_{\mathbf{p}}(E)}{\Delta \Sigma_{\mathbf{p}_{\|}}(E)} .
\end{aligned}
$$

To obtain the second equality in Eq. (13), we have used the following relation and definitions:

$$
\begin{aligned}
& R_{\mathrm{p}}^{+} R_{\mathrm{p}}^{-}=\left(R_{\mathrm{p}}^{+}-R_{\mathrm{p}}^{-}\right) /\left[\left(R_{\mathrm{p}}^{-}\right)^{-1}-\left(R_{\mathrm{p}}^{+}\right)^{-1}\right], \\
& \Delta R_{\mathrm{p}}=R_{\mathrm{p}}^{+}-R_{\mathrm{p}}^{-},
\end{aligned}
$$

and

$$
\left(R_{\mathrm{p}}^{-}\right)^{-1}-\left(R_{\mathrm{p}}^{+}\right)^{-1}=\Sigma_{\mathrm{p}_{\|}}^{+}-\Sigma_{\mathrm{p}_{\|}}^{-}=\Delta \Sigma_{\mathrm{p}_{\|}}
$$

By using the Einstein relation, $\sigma(E)=2 e^{2} \rho(E) D(E)$,

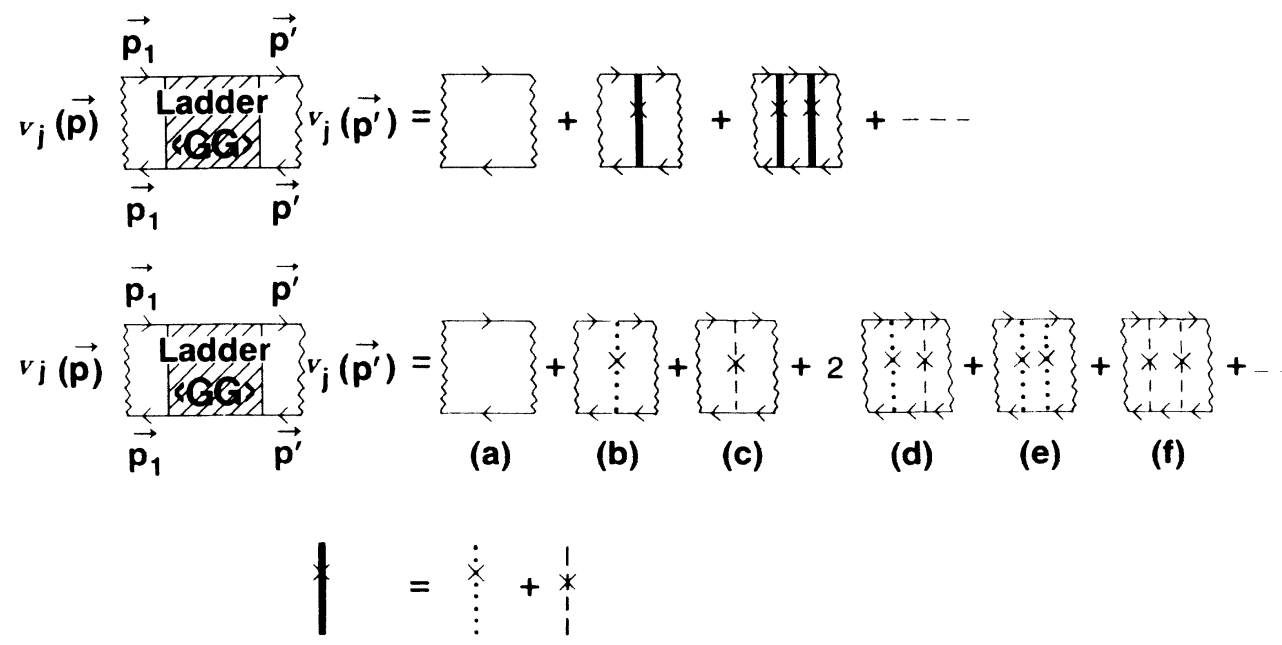

FIG. 1. Each vertex in the ladder diagrams (bold solid line) consists of two types of scattering. The dotted line denotes the 1D scattering with a strength of $\left[W^{2}(1-\theta)^{2} N_{\|} / 12\right] \delta_{\mathrm{q}_{\|}, 0}$. 3D isotropic scattering, denoted by a dash line, has the strength $W^{2} \theta^{2} / 12$. The solid line denotes the averaged Green's function $R$. 
where $\rho(E)$ is the density of states, the corresponding bare diffusion constant becomes

$$
D_{\perp}^{(0)}(E)=\frac{1}{2 \pi \rho N} \sum_{\mathbf{p}} \frac{v_{z}^{2}(\mathbf{p}) \Delta R_{\mathbf{p}}}{\Delta \Sigma_{\mathbf{p}_{\|}}},
$$

with

$$
\rho(E)=\frac{-1}{\pi} \operatorname{Im} \sum_{\mathrm{p}} R_{\mathrm{p}}^{+}(E)=\frac{i}{2 \pi N} \sum_{\mathrm{p}} \Delta R_{\mathrm{p}}(E) .
$$

For $\sigma_{\|}$, on the other hand, one can allow any number of 1D scatterings (dotted lines) in the diagram because they preserve the transverse momentum. The summation of this class of diagrams [Figs. 1(b), 1(e), etc.] is carried out in Appendix B. The final expressions for the bare conductivity and diffusion constant are

$$
\sigma_{\|}^{(0)}(E)=2 e^{2} \rho(E) D_{\|}^{(0)}(E)=\frac{6 e^{2} v_{\|}^{2}(E)}{\pi W^{2} \theta^{2}},
$$

$$
D_{\|}^{(0)}(E)=\frac{3 v_{\|}^{2}(E)}{\pi W^{2} \theta^{2} \rho(E)},
$$

with

$$
v_{\|}^{2}(E)=\sum_{\mathbf{p}}\left[v_{x}^{2}(\mathbf{p})+v_{y}^{2}(\mathbf{p})\right] \frac{\Delta R_{\mathrm{p}}}{\Sigma_{\mathbf{p}} \Delta R_{\mathbf{p}}} .
$$

It should be noted that, in the limit of $\theta \rightarrow 0, D_{\|}^{(0)}$ diverges but $D_{\perp}^{(0)}$ is finite as can be seen from Eqs. (20) and (17). This is exactly the desired property for a randomly layered system.

To study the localization behavior, a MCD has to be included in the vertex of Eq. (12). For the $\perp$ component, the vertex correction $\delta \phi_{z z}^{R A(1)}$ due to the first-order MCD is shown in Fig. 2(a). However, for the $\|$ component we also have to include the class of ladder diagrams that contains only the 1D scatterings, e.g., the diagrams $L_{1}$ and $L_{2}$ in Fig. 2(e) also have to be included. Thus, the general first-order MCD correction to $\delta \phi_{j j}^{R A(1)}$ can be written as

$$
\begin{aligned}
\delta \phi_{j j}^{R A(1)}(\omega, E)=\frac{1}{4 \pi^{2}} \sum_{\mathbf{p}, \mathbf{p}^{\prime}} v_{j}(\mathbf{p}) v_{j}\left(\mathbf{p}^{\prime}\right) R_{\mathbf{p}}^{+}\left(E_{+}\right) R_{\mathbf{p}}^{-}\left(E_{-}\right) R_{\mathbf{p}^{\prime}}^{+}\left(E_{+}\right) R_{\mathbf{p}^{\prime}}^{-}\left(E_{-}\right) \\
\times\left[\sum_{\mathbf{p}_{1}, \mathbf{p}_{2}} U_{j}^{(0)}\left(\mathbf{p}, \mathbf{p}_{1} ; \omega, E\right) U^{(M)}\left(\mathbf{p}_{1}, \mathbf{p}_{2} ; \omega, E\right) U_{j}^{(0)}\left(\mathbf{p}^{\prime}, \mathbf{p}_{2} ; \omega, E\right)\right],
\end{aligned}
$$

with

$$
U_{z}^{(0)}\left(\mathbf{p}, \mathbf{p}_{1} ; \omega, E\right)=\delta_{\mathbf{p}, \mathbf{p}_{1}}, \quad j=z
$$

and

$$
U_{i}^{(0)}\left(\mathbf{p}, \mathbf{p}_{1} ; \omega, E\right)=U^{(0)}\left(\mathbf{p}, \mathbf{p}_{1} ; \omega, E\right), \quad j=i=x \text { or } y,
$$

where $U^{(M)}$ and $U^{(0)}$ are the functions represented by the diagrams shown in Fig. 2. Following a derivation similar to that given in Appendix B, the function $U^{(0)}$ can be evaluated easily, yielding

$$
U^{(0)}\left(\mathbf{p}, \mathbf{p}_{1} ; 0^{+}, E\right)=\delta_{\mathbf{p}, \mathbf{p}_{1}}+\frac{i}{2 \pi N_{\perp}} \delta_{\mathbf{p}_{\|},\left(\mathbf{p}_{1}\right)_{\|}} \frac{(1-\theta)^{2}}{\theta^{2}} \frac{\Delta R_{\mathbf{p}_{1}}(E)}{\rho(E)} .
$$

Evaluation of the function $U^{(M)}$ is carried out in Appendix C. The dominant terms in the final expression are

$$
\begin{aligned}
U^{(M)}\left(\mathbf{p}_{1}, \mathbf{p}_{2} ; \omega, E\right) \cong\left(\frac{W^{2}}{12 N}\right)^{2}[ & \left(\theta^{2}+(1-\theta)^{2} \theta^{2} \frac{W^{2}}{12 N_{\perp}} F\left(\left(\mathbf{p}_{1}\right)_{\|},\left(\mathbf{p}_{2}\right)_{\|}, \mathbf{k}_{1} ; \omega, E\right)\right] \\
\times & {\left[\theta^{2}+(1-\theta)^{2} \theta^{2} \frac{W^{2}}{12 N_{\perp}} F\left(\left(\mathbf{p}_{2}\right)_{\|},\left(\mathbf{p}_{1}\right)_{\|}, \mathbf{k}_{1} ; \omega, E\right)\right] \phi^{(0)}(\mathbf{k} ; \omega, E) } \\
& \left.+(1-\theta)^{4} N_{\|}^{2} \boldsymbol{F}\left(\left(\mathbf{p}_{1}\right)_{\|}\left(\mathbf{p}_{2}\right)_{\|}, \mathbf{k}_{1} ; \omega, E\right) \delta_{\left.\left(\mathbf{p}_{1}\right)_{\|},\left(\mathbf{p}_{2}\right)_{\|}\right]}\right]
\end{aligned}
$$

where

$$
\begin{aligned}
& \mathbf{k}=\mathbf{p}_{1}+\mathbf{p}_{2}, \\
& \phi^{(0)}(\mathbf{k} ; \omega, E)=\frac{\sum_{\mathbf{p}_{3}}\left[R_{\mathrm{p}_{3+}}^{+}\left(E_{+}\right) R_{\mathrm{p}_{3-}}^{-}\left(E_{-}\right)\right] /\left[1-A\left(\left(\mathbf{p}_{3}\right)_{\|} ; \omega, E\right)\right]}{1-\frac{W^{2} \theta^{2}}{12 N} \sum_{\mathbf{p}_{3}}\left[R_{\mathrm{p}_{3+}}^{+}\left(E_{+}\right) R_{\mathrm{p}_{3-}}\left(E_{-}\right)\right] /\left[1-A\left(\left(\mathbf{p}_{3}\right)_{\|} ; \omega, E\right)\right]},
\end{aligned}
$$

with 


$$
\begin{aligned}
& \mathbf{p}_{3 \pm}=\mathbf{p}_{3} \pm \frac{\mathbf{k}}{2} \\
& A\left(\left(\mathbf{p}_{3}\right)_{\|} ; \omega, E\right)=\frac{W^{2}(1-\theta)^{2}}{12 N_{\perp}} \sum_{\left(\mathbf{p}_{4}\right)_{1}} R_{\left[\left(\mathbf{p}_{3}\right)_{\|}+\left(\mathbf{p}_{4}\right)_{\perp}\right]+}^{+}\left(E_{+}\right) R_{\left[\left(\mathbf{p}_{3}\right)_{\|}+\left(\mathbf{p}_{4}\right)_{1}\right]-}^{-}\left(E_{-}\right),
\end{aligned}
$$

and

$$
F\left(\left(\mathbf{p}_{1}\right)_{\|},\left(\mathbf{p}_{2}\right)_{\|}, \mathbf{k}_{1} ; \omega, E\right)=\frac{\sum_{\left(\mathbf{p}_{3}\right)_{1}} R_{\left(\mathbf{p}_{1}\right)_{\|}+\left(\mathbf{p}_{3}\right)_{1}+\mathbf{k}_{1} / 2}^{+}\left(E_{+}\right) R_{-\left(\mathbf{p}_{2}\right)_{\|}+\left(\mathbf{p}_{3}\right)_{\perp}-\mathbf{k}_{1} / 2}^{-}\left(E_{-}\right)}{1-\frac{W^{2}(1-\theta)^{2}}{12 N_{\perp}} \sum_{\left(\mathbf{p}_{3}\right)_{1}} R_{\left(\mathbf{p}_{1}\right)_{\|}+\left(\mathbf{p}_{3}\right)_{\|}+\mathbf{k}_{1} / 2}^{+}\left(E_{+}\right) R_{-\left(\mathbf{p}_{2}\right)_{\|}+\left(\mathbf{p}_{3}\right)_{1}-\mathbf{k}_{1} / 2}^{-}\left(E_{-}\right)} .
$$

It is shown in Appendix D that in the hydrodynamic limit $(\omega, k \rightarrow 0)$ the function $\phi^{(0)}$ has a $3 \mathrm{D}$ anisotropic diffusion pole of the form

$$
\phi^{(0)}(\mathbf{k} ; \omega, E) \cong \frac{2 \pi \rho(E) N}{-i \omega+D_{1}^{(0)}(E) k_{1}^{2}+D_{\|}^{(0)}(E) k_{\|}^{2}},
$$

with the bare diffusion constants $D_{\perp}^{(0)}$ and $D^{(0)}$ given by Eqs. (17) and (20). Thus, the first term of $U^{(M)}$ in Eq. (26) represents the coherent-backscattering effect due to the 3D scattering, which vanishes in the limit of $\theta \rightarrow 0$. The function $F$ in the last term of Eq. (26) also has the following simple expression in the hydrodynamic limit:

$$
F\left(\left(\mathbf{p}_{1}\right)_{\|},\left(\mathbf{p}_{1}\right)_{\|}, \mathbf{k}_{1} ; \omega, E\right) \cong \frac{\sum_{\left(\mathbf{p}_{3}\right)_{1}} \Delta R_{\left(\mathbf{p}_{1}\right)_{\|}+\left(\mathbf{p}_{3}\right)_{1}}(E)}{\mathrm{H}\left(\left(\mathbf{p}_{1}\right)_{\|}, \mathbf{k}_{1} ; \omega, E\right)}
$$

with

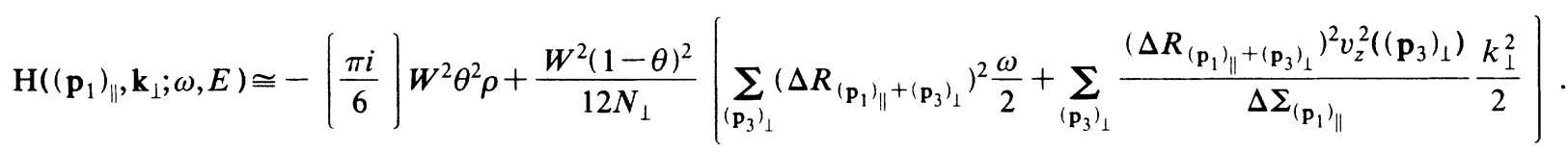

It is easily seen from Eqs. (33) and (34) that the function $F$ has a $\left(p_{1}\right)_{\|}$-dependent (1D) diffusion pole [last term of Eq. (34)] with an equivalent "inelastic" scattering time [first term of Eq. (34)]. The physics of this 1D inelastic scattering time will be discussed later. Thus the last term in $U^{(M)}$ of Eq. (26) represents solely the $1 \mathrm{D}$ coherent-backscattering effect in the layering direction, which vanishes in the isotropic limit of $\theta \rightarrow 1$. Since the 1D scattering preserves the transverse momentum, there is no such $1 \mathrm{D}$ coherent-backscattering effect for the transverse conductivity. Substituting the results of $U_{j}^{(0)}$ given in Eqs. (23)-(25) and $U^{(M)}$ given in Eqs. (26)-(34) into Eq. (22) yields, after some straightforward but lengthy manipulations, the first-order MCD corrections to the function $\phi_{j j}^{R A}$ :

$$
\delta \phi_{z z}^{R A(1)}(\omega, E)=\frac{1}{4 \pi^{2}} \sum_{\mathbf{p}} v_{z}^{2}\left(\mathbf{p}_{z}\right) \frac{\Delta R_{\mathrm{p}}}{\Delta \Sigma_{\mathbf{p}_{\|}}}\left(-\frac{\Delta R_{\mathbf{p}}}{\Delta \Sigma_{\mathbf{p}_{\|}}} B_{\perp}\left(\mathbf{p}_{\|} ; \omega, E\right)\right),
$$

with

$$
B_{\perp}\left(\mathbf{p}_{\|} ; \omega, E\right)=\left(\frac{W^{2}}{12 N}\right]\left[\theta^{4} \Delta \Sigma_{\mathbf{p}_{\|}}^{2} \sum_{\mathbf{k}} \frac{\phi^{(0)}(\mathbf{k} ; \omega, E)}{\mathrm{H}^{2}\left(\mathbf{p}_{\|}, \mathbf{k}_{1} ; \omega, E\right)}+(1-\theta)^{4} N_{\|}^{2} \sum_{\left(\mathbf{p}_{3}\right)_{\perp}} \Delta R_{\mathbf{p}_{\|}+\left(p_{3}\right)_{1}} \sum_{k_{\perp}} \mid \frac{1}{\mathbf{H}\left(\mathbf{p}_{\|}, \mathbf{k}_{\perp} ; \omega, E\right)}\right),
$$

and

$$
\delta \phi_{i i}^{R A(1)}(\omega, E)=\frac{3 i}{2 \pi^{3} \theta^{2} W^{2} \rho} \sum_{\mathbf{p}} v_{i}^{2}(\mathbf{p}) \Delta R_{\mathbf{p}}\left(\frac{i W^{2} \theta^{2} \Delta \Sigma_{\mathbf{p}_{\|}}}{12 \pi \rho N^{2}} \sum_{\mathbf{k}} \frac{\phi^{(0)}(\mathbf{k} ; \omega, E)}{\mathrm{H}^{2}\left(\mathbf{p}_{\|}, \mathbf{k}_{1} ; \omega, E\right)}\right) \quad(i=x \text { or } y),
$$

where $\phi^{(0)}$ and $\mathrm{H}$ are given by Eqs. (32) and (34). The correction of $\phi_{i j}^{(R A)}$ from the second-order MCD are shown in Fig. 3 for both the layering and the transverse directions. Again, for the transverse direction, the ladder diagrams $L_{1}$ and $L_{2}$, which contain only the 1D scatterings, should be included in $\delta \phi_{i i}^{R A(2)}$ because they give a nonzero contribution. The evaluation of these diagrams is rather lengthy but follows a procedure similar to that described in the evaluation of $\delta \phi_{j j}^{R A(1)}$ and will not be shown. The results are

$$
\delta \phi_{z z}^{R A(2)}=\frac{1}{4 \pi^{2}} \sum_{\mathbf{p}} v_{z}^{2}\left(\mathbf{p}_{z}\right) \frac{\Delta R_{\mathbf{p}}}{\Delta \Sigma_{\mathbf{p}_{\|}}}\left|-\frac{\Delta R_{\mathbf{p}}}{\Delta \Sigma_{\mathbf{p}_{\|}}} B_{\perp}\left(\mathbf{p}_{\|} ; \omega, E\right)\right|^{2}
$$


and

$$
\delta \phi_{i i}^{R A(2)}=\frac{3 i}{2 \pi^{3} \theta^{2} W^{2} \rho} \sum_{\mathbf{p}} v_{i}^{2}(\mathbf{p}) \Delta R \mathbf{p}\left(\frac{i W^{2} \theta^{2} \Delta \Sigma_{\mathbf{p}_{\|}}}{12 \pi \rho N^{2}} \sum_{\mathbf{k}} \frac{\phi^{(0)}(\mathbf{k} ; \omega, E)}{\mathbf{H}^{2}\left(\mathbf{p}_{\|} k_{\perp} ; \omega, E\right)}\right)^{2} \quad(i=x \text { or } y),
$$

where the function $B_{\perp}$ is given by Eq. (36). In fact, the generalization of the above results to the $n$th order MCD correction is simply to replace the power in the large parentheses of Eqs. (38) and (39) by $n$. It should be noted that in deriving the above results the following approximation has frequently been used:

$$
\left(\Delta R_{\mathrm{p}}\right)^{2}=\left(R_{\mathrm{p}}^{+}-R_{\mathrm{p}}^{-}\right)^{2} \cong-\left(R_{\mathrm{p}}^{+} R_{\mathrm{p}}^{-}+R_{\mathrm{p}}^{-} R_{\mathrm{p}}^{+}\right)=\frac{-2 \Delta R_{\mathrm{p}}}{\Delta \Sigma_{\mathrm{p}_{\|}}},
$$

where the small terms $R_{\mathrm{p}}^{+} R_{\mathrm{p}}^{+}$and $R_{\mathrm{p}}^{-} R_{\mathrm{p}}^{-}$have been neglected. This approximation may be generalized to

$$
\left(\Delta R_{\mathrm{p}}\right)^{n} \cong \Delta R_{\mathrm{p}}\left(-\frac{2}{\Delta \Sigma_{\mathbf{p}_{\|}}}\right)^{n-1}
$$

By summing the MCD corrections to infinite order and denoting the result as $\delta \phi_{i j}^{R A}$, the complex diffusion constant $D_{j j}(\omega, E)$ can be expressed as ${ }^{10,22}$

$$
\frac{D_{\perp}(\omega, E)}{D_{\perp}^{(0)}(E)}=1+\frac{\delta \phi_{z z}^{R A}}{\phi_{z z}^{R A(0)}}=\frac{1}{\sum_{\mathbf{p}} v_{z}^{2}\left(\mathbf{p}_{z}\right)\left(\Delta R_{\mathrm{p}} / \Delta \Sigma_{\left.\mathbf{p}_{\|}\right)}\right)} \sum_{\mathbf{p}} v_{z}^{2}(\mathbf{p}) \frac{\Delta R_{p}}{\Delta \Sigma_{\mathbf{p}_{\|}}} \frac{1}{1+\left(\Delta R_{\mathrm{p}} / \Delta \Sigma_{\mathbf{p}_{\|}}\right) B_{\perp}\left(\mathbf{p}_{\|} ; \omega, E\right)},
$$

and

$$
\begin{aligned}
\frac{D_{\|}(\omega, E)}{D_{\|}^{(0)}(E)} & =1+\frac{\delta \phi_{i i}^{R A}}{\phi_{i i}^{R A(0)}} \\
& =\frac{i}{2 \pi v_{\|}^{2} \rho(E) N} \sum_{\mathbf{p}} v_{\|}^{2}(\mathbf{p}) \Delta R_{\mathrm{p}} \frac{1}{1-\left(i W^{2} \theta^{2} \Delta \Sigma_{\mathbf{p}_{\|}} / 12 \pi \rho N^{2}\right) \sum_{\mathbf{k}}\left[\phi^{0}(\mathbf{k} ; \omega, E) / \mathrm{H}^{2}\left(p_{\|}, \mathbf{k}_{\perp} ; \omega, E\right)\right]},
\end{aligned}
$$

where $v_{\|}^{2}$ is defined by Eq. (21) and $\phi^{R A(0)}$ denotes the ladder diagram contributions to $\phi_{i j}^{R A}$, which can be obtained from Eqs. (11), (13), and (19).

In order to obtain the self-consistent equations to determine the mobility edge, approximations have to be imposed to simplify Eqs. (42) and (43) without losing the qualitative features of the problem. First, by using Eq. (41), the function of $\Delta R_{\mathrm{p}}$ in the denominator [1+( $\left.\left.\Delta R_{\mathrm{p}} / \Delta R_{\mathrm{p}}\right) B_{\perp}\right]$ of Eq. (42) can be approximated by $-2 / \Delta \Sigma_{\mathrm{p}_{\|}}$due to the existence of another $\Delta R_{\mathrm{p}}$ in the numerator under the summation of $p$. Thus both this factor and the corresponding one in Eq. (43) can be considered as functions of $\mathbf{p}_{\|}$only. Second, these factors can be decoupled from the summation of $\mathbf{p}$ by taking their averaged values, i.e., $\left\langle F\left(\mathbf{p}_{\|}\right)\right\rangle_{\mathbf{p}_{\|}}=1 / N_{\|} \Sigma_{\mathbf{p}_{\|}} F\left(\mathbf{p}_{\|}\right)$. In fact, this is not the only approximation one can choose. Alternatively, the average can also be defined by

$$
\left\langle F\left(\mathrm{p}_{\|}\right)\right\rangle_{\mathrm{p}_{\|}}=\Sigma_{\mathrm{p}} \Delta R_{\mathrm{p}} F\left(\mathrm{p}_{\|}\right) / \Sigma_{\mathrm{p}} \Delta R_{\mathrm{p}} .
$$

No qualitative difference in the final results has been found between these two approximations. In this work we choose the first average scheme. With the use of the above decoupling procedure, Eqs. (42) and (43) can be written respectively as

$$
\begin{aligned}
D_{\perp}(\omega, E)=D_{\perp}^{(0)}(E)+ & D_{\perp}(\omega, E)\left\langle\frac{2 B_{\perp}\left(\mathbf{p}_{\|}\right)}{\left(\Delta \Sigma_{\mathbf{p}_{\|}}\right)^{2}}\right\rangle_{\mathbf{p}_{\|}}, \\
D_{\|}(\omega, E)=D_{\|}^{(0)}(E)+ & D_{\|}(\omega, E) \frac{i W^{2} \theta^{2}}{12 \pi \rho N^{2}} \\
& \times\left\langle\Delta \Sigma_{\mathbf{p}_{\|}} \sum_{\mathbf{k}} \frac{\phi^{(0)}(\mathbf{k} ; \omega, E)}{\mathbf{H}^{2}\left(\mathbf{p}_{\|}, \mathbf{k}_{\perp} ; \omega, E\right)}\right\rangle_{\mathbf{p}_{\|}} .
\end{aligned}
$$

It should be noted that the function $B_{\perp}$ in Eq. (44) contains the previously mentioned $\mathbf{p}_{\|}$-dependent $1 \mathrm{D}$ diffusion pole with an inelastic scattering time as can be seen from the $1 / \mathrm{H}$ factor in Eqs. (36) and (34). In order to realize self-consistent procedure, it is required that an averaged diffusion constant with the form of $D_{\perp}^{(0)}(E)$ given by Eq. (17) should come out of this $1 \mathrm{D}$ diffusion pole. This can be realized if the function $\Delta \Sigma_{p_{\|}} H$ is approximated by its average. From Eq. (34), with the use of Eq. (41), it can be shown that

$$
\left\langle\Delta \Sigma_{\mathbf{p}_{\|}} \mathbf{H}\left(\mathbf{p}_{\|}, \mathbf{k}_{\|}, \omega, E\right)\right\rangle_{\mathbf{p}_{\|}} \cong \frac{-\pi W^{2}(1-\theta)^{2} \rho(E)}{6}\left(-i \omega+D_{\perp}^{(0)}(E) k_{\perp}^{2}+\frac{2 \theta^{2} \gamma(E)}{(1-\theta)^{2}}\right),
$$


where we have defined

$$
\gamma(E)=\frac{i}{2}\left\langle\Delta \Sigma_{\mathbf{p}_{\|}}\right\rangle_{\mathbf{p}_{\|}}
$$

Thus, in the final approximation, by averaging the function $\Delta \Sigma_{\mathbf{p}_{\|}} \mathbf{H}$ in Eqs. (44) and (45) separately, the following equations are obtained:

$$
\begin{aligned}
D_{\perp}(\omega, E)= & D_{\perp}^{(0)}(E)-\frac{1}{\pi \rho N} \sum_{\mathbf{k}} \frac{1}{\left(1+\left[(1-\theta)^{2} / 2 \gamma \theta^{2}\right]\left(-i \omega+D_{\perp}^{0} k_{\perp}^{2}\right)\right)^{2}} \frac{D_{\perp}(\omega, E)}{\left(-i \omega+D_{\perp}^{(0)} k_{\perp}^{2}+D_{\|}^{(0)} k_{\|}^{2}\right)} \\
& -\frac{1}{\pi \rho}\left(1-\frac{\tau_{\perp}}{\tau_{\|}}\right) \frac{1}{N_{\perp}} \sum_{\mathbf{k}_{1}} \frac{D_{\perp}(\omega, E)}{-i \omega+\left[2 \gamma \theta^{2} /(1-\theta)^{2}\right]+D_{\perp}^{(0)} k_{\perp}^{2}}, \\
D_{\|}(\omega, E)= & D_{\|}^{(0)}(E)-\frac{12 \gamma}{\pi^{2} \rho \theta^{2} W^{2} N} \sum_{\mathbf{k}} \frac{1}{\left\{1+\left[(1-\theta)^{2} / 2 \gamma \theta^{2}\right]\left(-i \omega+D_{\perp} k_{\perp}^{2}\right)\right\}^{2}} \frac{D_{\|}(\omega, E)}{\left(-i \omega+D_{\perp}^{(0)} k_{\perp}^{2}+D_{\|}^{(0)} k_{\|}^{2}\right)},
\end{aligned}
$$

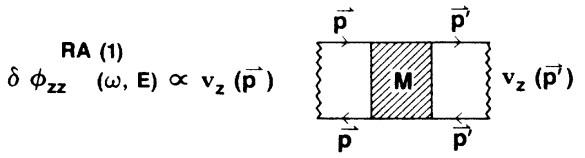

(a)

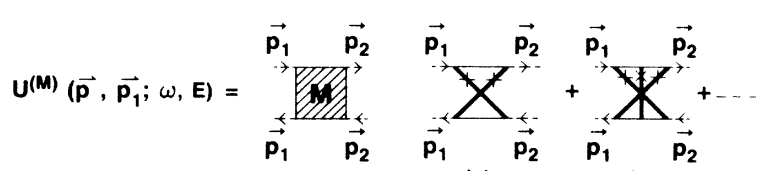

(b)

(c)

(d)

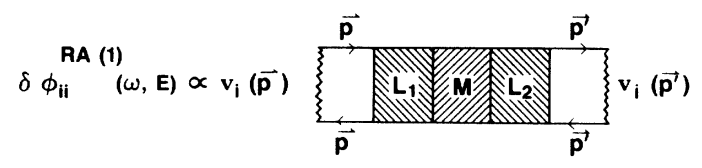

(e)

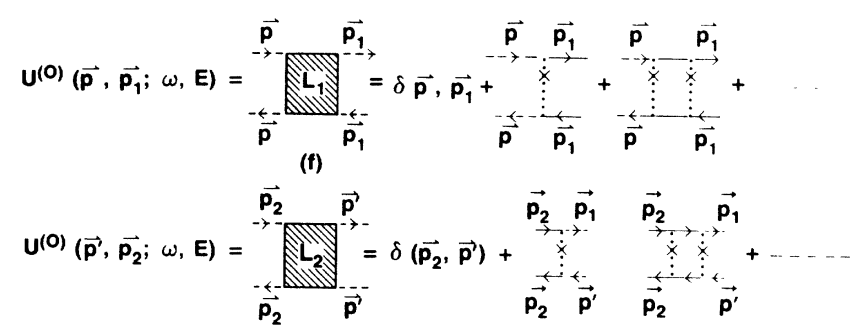

(g)

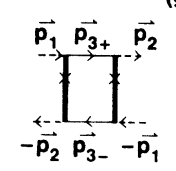

(h)

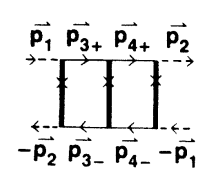

(i)
FIG. 2. (a) The first-order maximally crossed diagrams (MCD) contribution to the vertex correction $\phi_{z z}^{R A(1)}$ in the layering direction. (b) - (d) The MCD's that give rise to $U^{(M)}\left(\mathrm{p}_{1}, \mathrm{p}_{2} ; \omega, E\right)$, (e) for the transverse direction, the ladder diagrams $L_{1}$ and $L_{2}$ that give nonzero contribution are also included in the first-order MCD vertex correction $\delta \phi_{i i}^{R A(1)}$. (f) and (g) The ladder diagrams $L_{1}$ and $L_{2}$ that give rise to $U^{(0)}\left(\mathbf{p}, \mathbf{p}_{1} ; \omega, E\right)$ and $U^{(0)}\left(\mathbf{p}^{\prime}, \mathrm{p}_{2} ; \omega, E\right)$ respectively. (h) and (i) were obtained by redrawing the diagrams (c) and (d) in the particle-particle channel and then reversing the momentum of the lower lines. where $\tau_{\perp}$ and $\tau_{\|}$are respectively defined by

$$
\begin{aligned}
& \tau_{\perp}(E)=-i\left\langle\frac{1}{\Delta \Sigma_{\mathbf{p}_{\|}}}\right\rangle \mathbf{p}_{\|}, \\
& \tau_{\perp}(E)=\frac{6}{\pi W^{2} \theta^{2} \rho} .
\end{aligned}
$$

Here $\tau_{\perp}$ and $\tau_{\|}$are the relaxation times in the layering and transverse directions, respectively, as can be seen from Eqs. (17) and (20). From Eqs. (8) and (50), the inelastic scattering time $\tau_{\text {in }}\left[=(1-\theta)^{2} / 2 \gamma \theta^{2}\right]$ in the 1D diffusion pole of Eq. (48) can be roughly considered as the difference between the scattering times in the transverse and layering directions, which goes from zero to infinity as the anisotropy is increased. Physically, this is the time that limits the 1D diffusion process in the layering direction caused by the $3 \mathrm{D}$ isotropic scattering. The existence of $\tau_{\text {in }}$ thus provides a lower cutoff in $k_{\perp}$ space, i.e.,

$$
k_{\perp}^{(0)}(E)=\left(\frac{2 \gamma(E) \theta^{2}}{(1-\theta)^{2} D_{\perp}^{(0)}(E)}\right)^{1 / 2}
$$

below which the 1D diffusion becomes invalid. It should also be noted that Eqs. (48) and (49) give the correct $\theta \rightarrow 0$

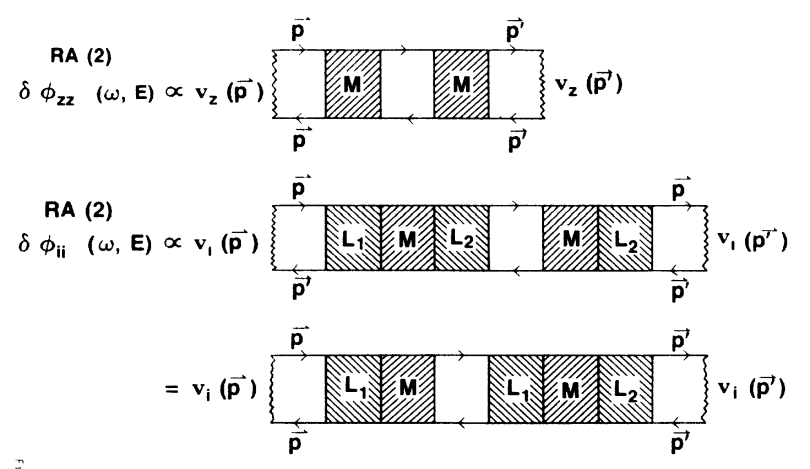

(a)

FIG. 3. The vertex correction $\delta \phi_{j j}^{R A(2)}$ due to the secondorder MCD's are shown for (a) the layering direction $\delta \phi_{z z}^{R A(2)}$ and (b) the transverse direction $\delta \phi_{i i}^{R A(2)} . M, L_{1}$, and $L_{2}$ represent the same classes of diagrams shown in Fig. 2. 
and $\theta \rightarrow 1$ limits. In the isotropic limit, $\theta \rightarrow 1$, it is easy to see from Eqs. (8) and (47) that $\tau_{\perp}=\tau_{\|}, D_{\perp}^{(0)}=D_{\|}^{(0)}$ $\left(=D^{(0)}\right)$, and $\gamma=\pi W^{2} \rho / 12$. Both Eqs. (48) and (49) then reduce to the standard $3 \mathrm{D}$ isotropic result; ${ }^{23}$ i.e.,

$$
D(\omega, E)=D^{(0)}(E)-\frac{1}{\pi \rho N} \sum_{\mathrm{p}} \frac{D(\omega, E)}{-i \omega+D^{(0)} k^{2}} .
$$

In the $1 \mathrm{D}$ randomly layering limit, $\theta \rightarrow 0$ we have $\tau_{\|} \rightarrow \infty$, while $\tau_{\perp}$ is finite. In this limit 3D diffusion terms in Eqs. (48) and (49) vanish, and Eq. (48) reduces to the standard $1 \mathrm{D}$ result in the layering direction.

\section{Mobility edge}

For a self-consistent theory, the bare diffusion constants $D_{\perp}^{(0)}$ and $D_{\|}^{(0)}$ in both the 3D and 1D diffusion poles of Eqs. (48) and (49) are replaced by $D_{\perp}(\omega, E)$ and $D_{\|}(\omega, E)$, respectively. However, in the case of a $1 \mathrm{D}$ diffusion pole, instead of using a lower cutoff $k_{1}^{0}$, we choose to first write the 1D pole in the form

$$
\left[-i \omega+D_{\perp}^{(0)}\left(\left(k_{\perp}^{0}\right)^{2}+k_{\perp}^{2}\right)\right]^{-1}
$$

with $k_{\perp}^{(0)}$ defined by Eq. (51) and then renormalize as $D_{\perp}^{(0)}$ afterwards. This has the equivalent effect of introducing a lower cutoff. The mobility edge equations are obtained by requiring $D_{1}\left(\omega=0^{+}, E^{*}\right)=D_{\|}\left(\omega=0^{+}, E^{*}\right)=0$ :

$$
\begin{aligned}
\frac{D_{\perp}\left(0^{+}, E^{*}\right)}{D_{\perp}^{(0)}\left(E^{*}\right)}=0=1- & \frac{F_{\perp}\left(E^{*}\right) r\left(E^{*}\right)}{N} \\
& \times \sum_{\mathbf{k}} \frac{1}{r\left(E^{*}\right) k_{\perp}^{2}+k_{\|}^{2}}-Q_{0}\left(E^{*}\right)
\end{aligned}
$$

and

$$
\frac{D_{\|}\left(0^{+}, E^{*}\right)}{D_{\|}^{(0)}\left(E^{*}\right)}=0=1-\frac{F_{\|}\left(E^{*}\right)}{N} \sum_{\mathrm{k}} \frac{1}{r\left(E^{*}\right) k_{\perp}^{2}+k_{\|}^{2}},
$$

with

$$
\begin{aligned}
& F_{\perp}(E)=\frac{1}{\pi \rho(E) D_{\perp}^{(0)}(E)}, \\
& r(E)=\frac{D_{\perp}\left(0^{+}, E\right)}{D_{\|}\left(0^{+}, E\right)},
\end{aligned}
$$

$$
Q_{0}(E)=\left\lfloor 1-\frac{\tau_{\perp}(E)}{\tau_{\|}(E)}\right) \frac{F_{\perp}(E)}{N} \sum_{k_{\perp}} \frac{1}{\left[k_{\perp}^{(0)}(E)\right]^{2}+k_{\perp}^{2}},
$$

and

$$
F_{\|}(E)=\frac{4 \gamma(E)}{\pi \rho(E) v_{\|}^{2}(E)},
$$

where Eq. (20) has been used to obtain Eq. (58). The function $Q\left(E^{*}\right)$ in Eq. (53) is due to the 1D scattering. Although both $D_{\perp}$ and $D_{\|}$vanish at the mobility edge, their ratio $r\left(E^{*}\right)$ is finite and, from Eqs. (53) and (54), has the following expression:

$$
r\left(E^{*}\right)=\frac{F_{\|}\left(E^{*}\right)}{F_{\perp}\left(E^{*}\right)}\left[1-Q_{0}\left(E^{*}\right)\right]
$$

Substituting Eq. (59) into Eq. (53) or (54) yields a single equation for the mobility edge as

$$
\begin{aligned}
1= & F_{\|}\left(E^{*}\right) F_{\perp}\left(E^{*}\right) \frac{1}{N} \\
& \times \sum_{\mathbf{k}} \frac{1}{F_{\perp}\left(E^{*}\right) k_{\|}^{2}+F_{\|}\left(E^{*}\right)\left[1-Q_{0}\left(E^{*}\right)\right] k_{\perp}^{2}} .
\end{aligned}
$$

The summation over $\mathbf{k}$ represents a double integral over $\mathbf{k}_{\|}$and $\mathbf{k}_{1}$, each having a upper cutoff of $k_{\|}^{c}=\chi_{0} / l_{\|}\left(E^{*}\right)$ and $k_{\perp}^{c}=\chi_{0} / l_{1}\left(E^{*}\right)$, where $l_{\|}$and $l_{\perp}$ are the mean free paths in the transverse and layering directions given by $l_{\|}=3 \sqrt{2} D_{\|}^{(0)} / v_{\|}$and $l_{\perp}=3 D_{\perp}^{(0)} / v_{\perp}$. The arbitrary constant $\chi_{0}$ is of order unity and is chosen to be 0.40 so that we recover the known critical value of $W_{c} / t=16.2$ in the isotropic limit $\theta=1, E=0$. Equation (60) may be solved numerically as a function of $\theta$ at various Fermi energies. Results are presented and discussed in Sec. IV.

\section{Localization lengths}

In the localized regime, the equations for the localization lengths $\xi_{\perp}$ and $\xi_{\|}$in the layering and transverse directions can be obtained from Eqs. (48) and (49) by first renormalizing the diffusion constants and then setting

$$
\lim _{\omega \rightarrow 0}\left[-i \omega / D_{\perp, \mid}(\omega, E)\right]=\xi_{\perp, \|}^{-2}(E) .
$$

If we define the function $r(E)$ as the square of the ratio of localization lengths $\left(\xi_{1, \|}\right)^{2}$, the localization length equations become

$$
1=F_{\perp}(E) r(E) \frac{1}{N} \sum_{\mathbf{k}} \frac{1}{r(E)\left[\xi^{-2}(E)+k_{\perp}^{2}\right]+k_{\|}^{2}}-F_{\perp}(E)\left(1-\frac{\tau_{\perp}(E)}{\tau_{\|}(E)}\right) \frac{1}{N_{\perp}} \sum_{\mathbf{k}_{\perp}} \frac{1}{\xi_{\perp}^{2}(E)+\left[k_{\perp}^{0}(E)\right]^{2}+k_{\perp}^{2}},
$$

and

$$
1=\frac{F_{\|}(E)}{N} \sum_{\mathbf{k}} \frac{1}{r(E)\left[\xi_{\perp}^{-2}(E)+k_{\perp}^{2}\right]+k_{\|}^{2}} .
$$

In general, Eqs. (61) and (62) have to be solved numerically. However, at small randomness the asymptotic behav- ior of $\xi_{\perp}$ and $\xi_{\|}$may be studied analytically. This is done at the end of Sec. IV.

\section{NUMERICAL APPROACH}

Since a significant amount of approximations have been introduced in obtaining the analytical equations on 
the mobility edge and the localization lengths, the validity and accuracy of the analytic predictions have to be verified and checked by numerical simulations. The numerical method used to determine the mobility edge is the standard finite-size scaling method. ${ }^{24}$ Consider a disordered system governed by the Hamiltonian defined through Eqs. (1)-(3) in a rod geometry of length $N$ and cross section $\boldsymbol{M} \times \boldsymbol{M}$. The layering direction is defined to be the lengthwise direction along the rod. Both ends of the rod are connected to perfect leads, i.e., $W=0$. For such a system the transmission amplitude of an electron through the disordered rod can yield the localization length $L_{M}$ of the rod.

If the periodic boundary condition is chosen in the transverse direction, then the propagating eigenstates in the ordered region are just plane waves labeled by a continuous momentum index $K_{z}$ in the direction along the rod and two discrete transverse momentum indices $K_{x}$ and $K_{y}=\pi n /(M+1) \quad(n=0, \pm 1, \ldots, \pm M / 2$ if $M$ is even; $n= \pm 1, \pm 2, \pm(M+1) / 2$ if $M$ is odd). The dispersion relation is then

$$
\frac{E}{t}=2\left(\cos K_{z}+\cos K_{x}+\cos K_{y}\right) \text {. }
$$

Consider each set $\left(K_{x}, K_{y}\right)$ as a channel, denoted as $I$. There are a total of $M \times M$ channels in the rod. For a given energy $E / t$, the real solutions of $K_{z}$ from Eq. (63) determine the number of channels open for wave propagation. The transmission matrix $t_{I J}$ between a channel $I$ at one end of the rod and a channel $J$ at the other end is equivalent to Green's function $G_{I J}(0, N)$, which can be calculated by using the recursive Green's-function technique as described in the work of Lee and Fisher. ${ }^{4}$ Here we simply extend the method to the $3 \mathrm{D}$ case. The localization length $L_{M}$ of the rod is related to the transmission matrix by

$$
L_{M}=\frac{-1}{2 N} \ln \operatorname{Tr} t^{\dagger} t
$$

The length $N$ has to be long enough so that $L_{M}$ has converged to a constant value. In addition, the variance $\Delta L_{M}(N)^{2}$ has to be small so that the value of $L_{M}$ is accurate. This can be achieved either by averaging over many configurations or by considering a long rod. From the knowledge of $L_{M}$ the localization character of a state is determined by the behavior of $L_{M} / M$ versus $M$ as described by MacKinnon and co-workers; ${ }^{24}$ i.e., $L_{M} / M$ increasing with $M$ indicates a delocalized state, whereas $L_{M} / M$ decreasing with $M$ indicates a localized state.

\section{RESULTS AND DISCUSSIONS}

\section{A. Mobility edge curves}

In the numerical calculations based on Eq. (60) the mobility edge $W_{c}(\theta) / t$ is calculated as a function anisotropy $\theta$ for each fixed value of the Fermi energy. Since the system is symmetric in $E$, we only need to study the portion $E>0$ regime. The Fermi energies chosen for numerical evaluations are $E / t=0,2,4,5,6$, and 7 . The corre- sponding curves of $W_{c}(\theta) / t$ are shown by solid curves in Figs. $4(\mathrm{a})-4(\mathrm{f})$. The interesting feature about these curves is that for each $E / t$ there is a finite critical value of $\theta=\theta_{c}$ below which the electron is localized for any randomness. The value of $\theta_{c}$ increases from 0.18 at the band center $(E / t=0)$ to unity when the band edge $(E / t)$ is approached. The mathematical origin of this $\theta_{c}$ comes from the function $Q_{0}$ in Eq. (53). When the randomness is small, it is easy to see from Eqs. (55) and (58) that both functions $F_{\perp}$ and $F_{\|}$contain a factor $W^{2}$. However, by using the upper cutoff $k_{1, \|}^{c}=\chi_{0} / l_{1, \|}$ discussed in Sec. II, the $\mathbf{k}$ space and $k_{1}$ space summation in 3D and 1D diffusion poles of Eqs. (53) and (54) give additional factors $W^{2}$ and $W^{-2}$, respectively. Thus, as $W \rightarrow 0^{+}$, the $3 \mathrm{D}$ localization effect disappears as expected, but the 1D localization term $Q_{0}$ becomes independent of $W$ and is only a function of $\theta$. In fact, the value of $Q_{0}$ goes montonically from zero when $\theta=1$ to infinity when $\theta=0$ as can be seen from the inelastic scattering length term, $\left(k_{\perp}^{0}\right)^{2}$, in Eq. (57). The critical $\theta_{c}$ is then determined by the condition that $Q_{0}$ has the value unity. Physically, the existence of this nonzero $\theta_{c}$ can also be understood from this inelastic scattering term. In the randomly layering limit $(\theta=0)$, the only scattering is the $1 \mathrm{D}$ scattering in the layering direction and the system is effectively $1 \mathrm{D}$ with a localization length $\xi_{\perp}$, proportional to $W^{-2}$. If the isotropic scattering is turned on $(\theta \neq 0)$, then the inelastic scattering length, $\left(k^{0}\right)^{-1}$, which is also proportional to $W^{-2}$, becomes finite due to the factor $(1-\theta) / \theta$ in Eq. (51). If $\theta$ is small such that the inelastic scattering length is larger than some effective 1D localization length, then the electrons cannot feel the $3 \mathrm{D}$ scattering and remain localized. The critical $\theta_{c}$ is thus the value of $\theta$ at which the above two lengths become comparable and the system loses its $1 D$ character. The same physical interpretation of $\theta_{c}$ emerges from the localization length analysis at the end of this section. It should also be noted that this 1D localization effect becomes increasingly important as the band edge $(E / t=6)$ is approached, where the density of states $\rho(E)$ in Eq. (55) reduces to zero. This is the reason why $\theta_{c}$ increases as $E$ moves away from the band center.

Above $\theta_{c}$, the value of $W_{c} / t$ is seen from Fig. 4 to have a peak that is above the $3 \mathrm{D}$ isotropic value. This is due to the net decrease of isotropic disorder, for a fixed $W$, as $\theta$ decreases from 1 . As a result, $W_{c}$ increases with decreasing $\theta$ until the $1 \mathrm{D}$ scattering catches up, at which point $W_{c}$ plunges to zero rather abruptly. Another interesting effect is noted to exist for $E / t \simeq 5-6$ [Figs. 4(d) and $4(\mathrm{e})$ ], i.e., close to the band edge. Here $W_{c}(\theta)$ is seen to form a loop so that in a range of $\theta$ values there can be two values of $W_{c}$ for a given $\theta$. This reentry behavior may be understood as follows. Since the density of states is small near the band edge, the effect of increasing $W$ is to increase the density of states and to reduce the localization effect. ${ }^{25}$ Once the states become extended, further increasing of $W$ is then to enhance the $3 \mathrm{D}$ coherentbackscattering effect and, hence, induce localization again. This is similar to the reentry behavior seen in the $E / t>6$ region of the isotropic system ${ }^{6,6,24,25}$ except that the abscissa is $E / t$ rather than $\theta$. Figure $4(\mathrm{f})$ shows the 
mobility edge curve for $E / t=7$. Again the reentry behavior exists. However, the lower $W_{c}$ curve now ends at some finite value of $W_{c} / t \simeq 7.0$ as $\theta \rightarrow 1$, which recovers the reentry behavior of an isotropic model for $E / t>6$.

The results presented above are obtained from di- agrammatical analysis and are by no means rigorous. We have also carried out numerical simulations by using the finite-size scaling method described in the preceding section. For any chosen cross section $M \times M$, the transmission matrix $t$ is calculated for a rod of $N$ sites in length.
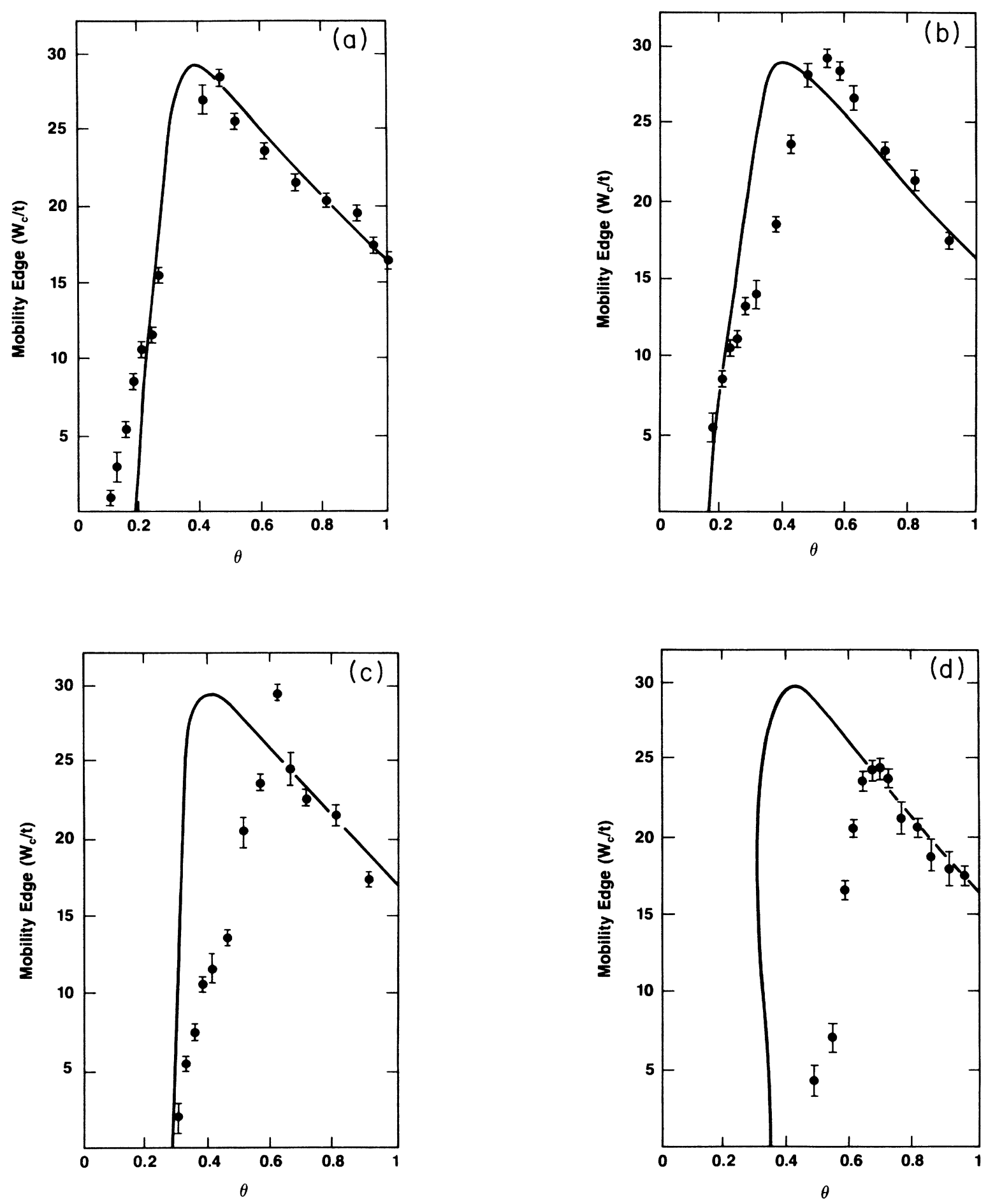

FIG. 4. Values of $W_{c} / t$ plotted as a function of $\theta$ for (a) $E / t=0$, (b) $E / t=2$, (c) $E / t=4$, (d) $E / t=5$, (e) $E / t=6$, and (f) $E / t=7$. The solid lines denote the results calculated from diagrammatical analysis. Simulation results are denoted by dots. 

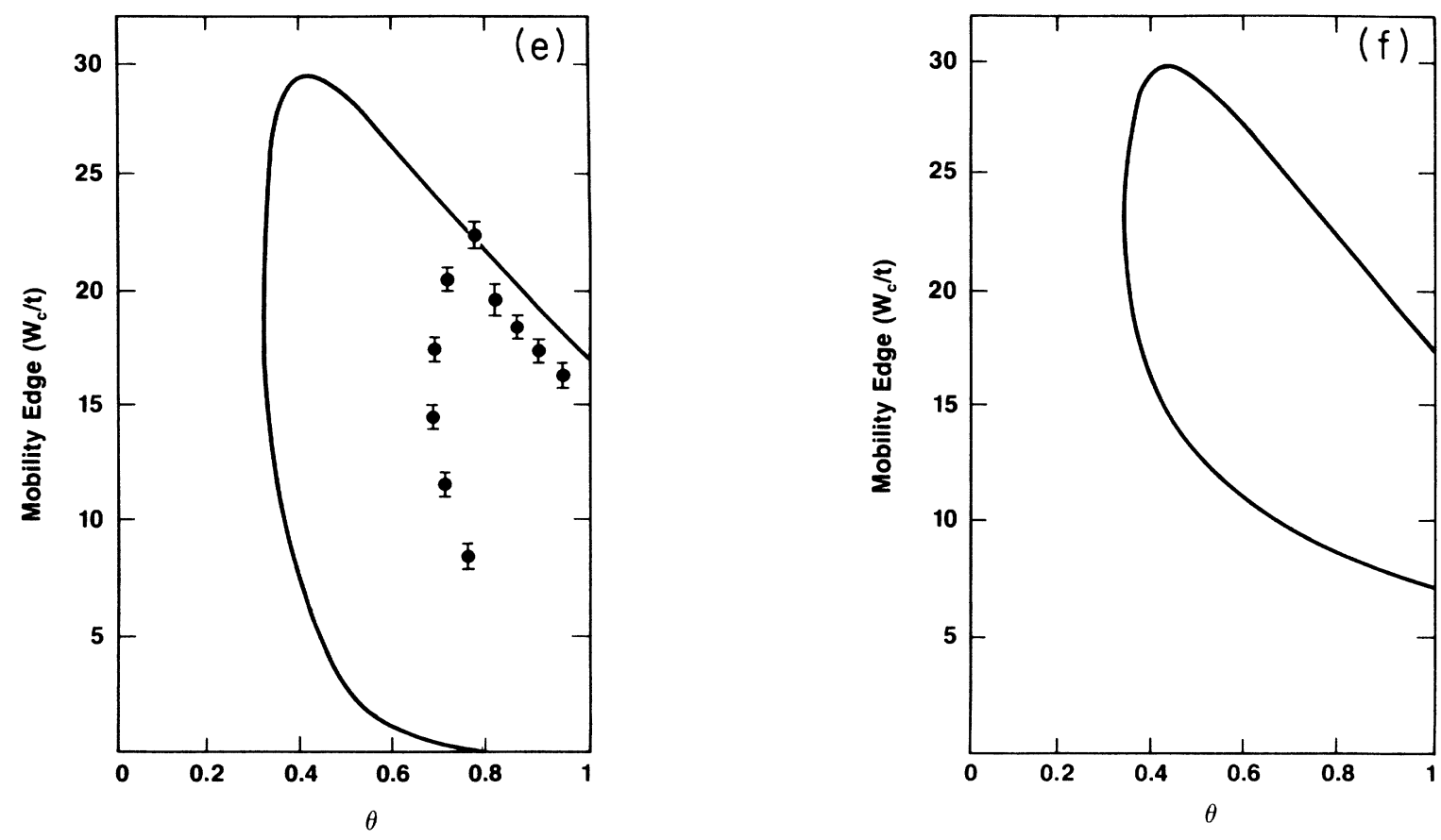

FIG. 4. (Continued).

The localization length $L_{M}$ is then obtained by using Eq. (64). The slope of the curve $L_{M} / M$ versus $M$ determines the nature of the states. In our numerical calculations, the value of $M$ runs from 2 to 8 and $N$ up to $4 \times 10^{4}$. In Fig. 5, a typical result is shown for $E / t=0, \theta=0.4$. The behavior of $L_{M} / M$ is plotted as a function of $M$ for vari-

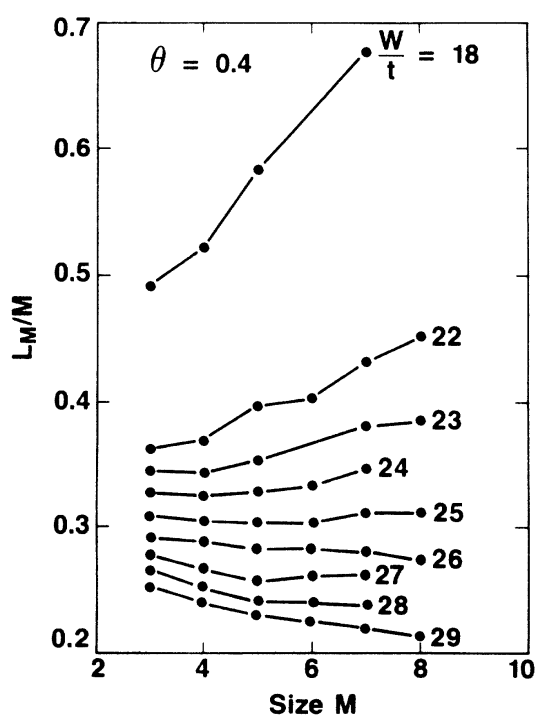

FIG. 5. Behavior of $L_{M} / M$ plotted as a function of $M$ for $E / t=0, \theta=0.4$, and various values of $W / t$. Curves with positive slopes denote delocalized states, and curves with negative slopes denote localized states. The mobility edge in this case is located between $W / t=26$ and 29 . ous values of $W$. The curves with positive slopes denote delocalized states, and the curves with negative slopes denote localized states. Mobility edge in this case is located between $W_{c} / t \approx 26$ and 29 . From a collection of these curves the approximate values of $W_{c} / t$ are obtained as a function of $E / t$ and $\theta$. These are shown by dots in Figs. 4(a)-4(e) together with the analytical curves. It is seen that for $E / t=0$ [Fig. 4(a)] the agreement with numerical results is remarkably good. Both the peak value of $W_{c} / t$ as well as the sharp rise of $W_{c} / t$ beyond $\theta_{c}$ are well reproduced. The value of $\theta_{c} \simeq 0.18$ is also in reasonable agreement with the extrapolated numerical value of $\theta_{c} \simeq 0.1$ The reentry behavior is also seen at $E / t=5$ and 6 [Fig. 4(d) and 4(e)]. Since the density of states is small near the band edge, large fluctuations prevent us from getting any reliable result when $W / t$ is small. However, both analytic and numerical results give consistent qualitative characteristics of this model, e.g., the existence of $\theta_{c}$, the reentry behavior, etc. The quantitative agreement is noted to deteriorate as $E / t$ increases towards the band edge. This is due to various approximations used in the theory. For instance, the third term for $U^{(M)}$ of Eq. (C18), which has been ignored, might become important in comparison with the second term when $\theta \rightarrow 1$. The inclusion of this term could potentially enhance the 1D localization effect (or suppress the extended region), thereby giving a closer agreement between the analytical and numerical results in the region near the band edge.

The existence of a finite $\theta_{c}$ is not found in the previously studied anisotropic models. ${ }^{16-20}$ In these models the anisotropy comes from either the scattering potential or the effective masses, while the site energy remains isotro- 
pic and random. It has been shown that, in the metallic limit, all these models can be reduced to the singleparameter scaling theory by making a suitable anisotropic scale change. Therefore, the system exhibits 3D Anderson transition as soon as it deviates from strict one (or two) dimensionality. In particular, this has been shown explicitly for the anisotropic hopping model, where an appreciable amount of disorder is always required to localize the $E / t=0$ state in a highly quasi-two-dimensional system. ${ }^{20}$ In contrast, the anisotropy in our model is due to the infinite correlation of randomness in each layer. This infinite correlation gives rise to an effective 1D scattering in the layering direction that conserves the momentum in the transverse directions. Since this 1D scattering provides a coherent-backscattering effect only in the layering direction but not in the transverse directions, distinct features from the previously studied anisotropic model hopping emerge. Also, because of the presence of the $1 \mathrm{D}$ diffusion pole in the layering direction [Eq. (48)] our problem is not reducible to a singleparameter scaling theory even in the metallic limit. Therefore, there exists no anisotropic scaling that can reduce our model to an isotropic random model.

\section{B. Localization lengths}

In the localized region, the localization lengths $\xi_{\perp}$ and $\xi_{\|}$can be obtained in general by solving Eqs. (61) and (62). However, in the case of small randomness, their asymptotic behaviors can be obtained as follows. Given fixed $E / t(E / t<6)$ to the leading order in $W$, Eqs. (51), (55), and (58) can be written as

$$
\begin{aligned}
& k_{\perp}^{(0)} \cong h(\theta) W^{2} \\
& F_{\perp} \cong f_{\perp}(\theta) W^{2} \\
& F_{\|} \cong f_{\|}(\theta) W^{2}
\end{aligned}
$$

We can also write the momentum cutoff as

$$
k_{\perp}^{c}=\frac{\chi_{0}}{l_{\perp}} \cong h_{\perp}(\theta) W^{2}
$$

and

$$
k_{\|}^{c}=\frac{\chi_{0}}{l_{\|}} \cong h_{\|}(\theta) W^{2} .
$$

From Eqs. (61) and (62), the following asymptotic forms for $\xi_{\perp}$ and $\xi_{\|}$can be inferred:

$$
\xi_{\perp} \cong \frac{c(\theta)}{W^{2}}
$$

and

$$
\xi_{\|} \cong g(\theta, W) \exp \left(\frac{d(\theta)}{W^{4}}\right) .
$$

Here the $W$ dependence of the function $g(\theta, W)$ is a power form. Equations (70) and (71) can be proved by inserting them into Eqs. (61) and (62). After integration, it is found that

$$
1 \cong \frac{f_{\perp} h_{\perp} d c^{2}}{4 \pi^{2} W^{2} g^{2}} \exp \left(\frac{-2 d}{W^{4}}\right)+\frac{f_{\perp} \Delta}{\pi\left(h^{2}+c^{-2}\right)^{1 / 2}}
$$

and

$$
1 \cong \frac{f_{\|} h_{\perp} d}{4 \pi^{2}}
$$

with

$$
\Delta=\tan ^{-1}\left(\frac{h_{\perp}}{\left(h^{2}+c^{-2}\right)^{1 / 2}}\right) .
$$

By neglecting the first term on the right hand side of Eq. (72), Eqs. (72) and (73) for $c(\theta)$ and $d(\theta)$ can be solved and are indeed found to be $W$ independent. Higher-order estimation from Eq. (62) suggests that $g(\theta, W)$ behaves like $h_{\|}^{-1} W^{-2}$. Thus Eq. (70) gives the same asymptotic behavior as the $1 \mathrm{D}$ result. However, Eq. (71) gives a behavior that is different from the standard 2D result of $\xi_{2 \mathrm{D}} \cong\left(a_{\|} / W^{2}\right) \exp \left(a^{2} / W^{2}\right) .^{26}$

Finally, we analyze how $c(\theta)$ changes when the mobility edge is approached along a constant but small $W / t$. By neglecting the 3D localization term in Eq. (61), the remaining terms describe an effective 1D localization problem with an effective localization length $\xi_{\perp}^{e}=\left[\left(k_{\perp}^{0}\right)^{2}+\xi_{\perp}^{-2}\right]^{1 / 2}$. The function $\xi_{\perp}^{e}$ can also be written in the form

$$
\xi_{\perp}^{e} \cong \frac{b(\theta)}{W^{2}},
$$

with

$$
b^{-2}(\theta)=h^{2}(\theta)+c^{-2}(\theta)
$$

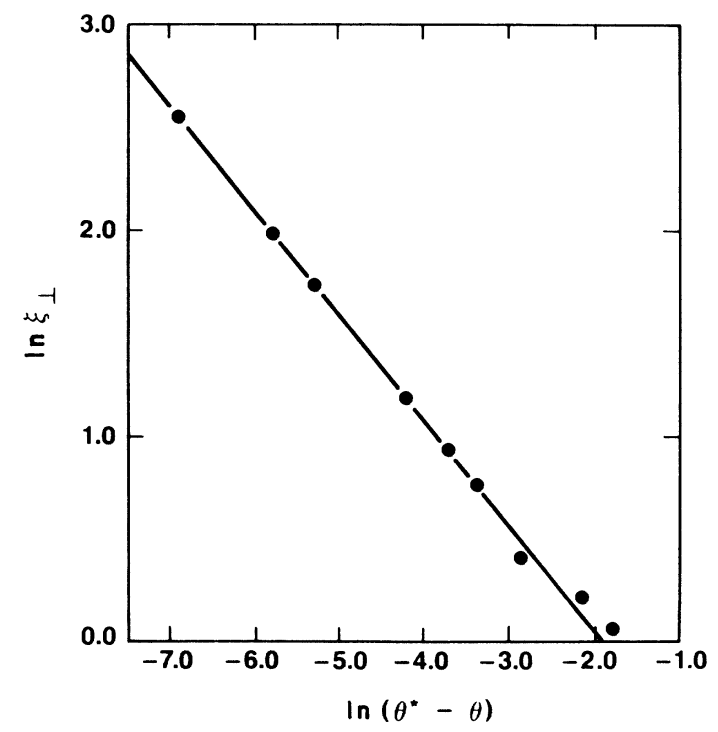

FIG. 6. At $E / t=0, W / t=10$, the localization length as a function of $\theta$ in the layering direction $\xi_{1}(\theta)$ as obtained by solving Eqs. (61) and (62) (denoted by the dots). The slope in this $\log -\log$ plot shows that $\xi_{1}(\theta)$ diverges with an exponent $\frac{1}{2}$ near the mobility edge $\theta^{*}=0.215$. 
or

$$
c(\theta)=\left[b^{-2}(\theta)-h^{2}(\theta)\right]^{-1 / 2} .
$$

Since $h^{-1}(\theta)$ and $b(\theta)$ are, respectively, the quantities that measure the inelastic scattering time and effective 1D localization length, $b$ is always smaller than $h^{-1}$ in the localized region so that $c$ is real and positive. As the mobility edge $\theta^{*}\left(\theta^{*} \rightarrow \theta_{c}\right.$ as $\left.W \rightarrow 0\right)$ is approached, two lengths become closer and $c$ diverges. The expansion of $c(\theta)$ near $\theta^{*}$ gives an exponent $\frac{1}{2}$, i.e., $c(\theta) \propto\left(\theta-\theta^{*}\right)^{-1 / 2}$. In order to verify this exponent, we have numerically calculated $\xi_{\perp}$ and $\xi_{\|}$from Eqs. (61) and (62) for the case $E / t=0, W / t=10$, and $\theta^{*}=0.215$. In Fig. 6 we show the log-log plot of $\xi_{1}(\theta)$ versus $\theta-\theta^{*}$. The slope is indeed found to be exactly $-\frac{1}{2}$.

\section{CONCLUDING REMARKS}

In this work, an anisotropically disordered model is proposed where the site energy randomness is anisotropic and intermediate between different dimensions. Both diagrammatical and numerical methods were used to study its localization characteristics. The predicted phase diagrams show many new features not found in previously studied anisotropic hopping models. At small randomness, the localization analysis in the localized region shows a typical 1D behavior in the layering direction. However, the behavior in the transverse direction is distinctly different from the standard 2D result. A critical value of the anisotropy is found above which the system makes the transitional 3D characteristics.

While our calculations are for the electronic tightbinding model, we expect the qualitative features of the results to be valid for classical waves as well. ${ }^{27}$ This means, on the one hand, that in predominantly layered systems (such as the Earth's subsurface) a small amount of lateral inhomogeneities would not modify the qualitative behavior of wave localization. ${ }^{28,29}$ Yet, on the other hand, the deliberate control of lateral inhomogeneities could offer a new handle not only on the 1D localization length, but also on the observation of localizationdelocalization transition. For example, if one can introduce a controlled amount of lateral inhomogeneities into random superlattices, then the delocalization of electronic states may be monitored by a change of sign in the temperature coefficient of resistivity. Similar experimental possibilities exist for classical wave propagation through randomly layered systems with transverse inhomogeneities. Related theoretical issues are under further study.

\section{APPENDIX A}

The derivation of Eq. (8), the self-energy expression in the SCBA, is given here. Denoting $\alpha=(i, m)$ and $\beta=(j, n)$, it is easily shown that

$$
\begin{aligned}
\left\langle\xi_{\alpha} \xi_{\beta}\right\rangle= & \left\langle\left[\theta \eta_{i m}+(1-\theta) \gamma_{m}\right]\left[\theta \eta_{j n}+(1-\theta) \gamma_{n}\right]\right\rangle_{c} \\
= & \left\{\begin{array}{l}
\frac{W^{2}}{12}\left[\theta^{2}+(1-\theta)^{2}\right] \text { if } \alpha=\beta \quad(i=j, m=n), \\
\frac{W^{2}}{12}(1-\theta)^{2} \text { if } \alpha \neq \beta \text { but } m=n, \\
0 \text { if } \alpha \neq \beta, m \neq n .
\end{array}\right.
\end{aligned}
$$

By defining $S(\mathbf{q})=N\langle v(\mathbf{q}) v(-\mathbf{q})\rangle_{c}$ and using Eqs. (5) and (6), one gets

$$
\begin{aligned}
& S(\mathbf{q})=N\langle v(\mathbf{q}) v(-\mathbf{q})\rangle_{c} \\
& =\frac{1}{N} \sum_{\alpha, \beta}\left\langle\varepsilon_{\alpha} \varepsilon_{\beta}\right\rangle_{c} e^{i \mathbf{q} \cdot\left(\mathbf{R}_{\alpha}-\mathbf{R}_{\beta}\right)} \\
& =\frac{W^{2}}{12}\left[\left[\theta^{2}+(1-\theta)^{2}\right]\right. \\
& \left.+\frac{(1-\theta)^{2}}{N} \sum_{l \neq j, m} e^{i \mathbf{q} \cdot\left(\mathbf{R}_{l m}-\mathbf{R}_{\jmath m}\right)}\right),
\end{aligned}
$$

where the first term comes from the contribution when $\alpha=\beta$, while the second term comes from the contribution from $\alpha \neq \beta$ but lying in the same layer. Since

$$
\sum_{\mathbf{R}_{\|} \neq 0} e^{i \mathbf{q} \cdot \mathbf{R}_{\|}}=\sum_{\mathbf{R}_{\|}} e^{i \mathbf{q} \cdot \mathbf{R}_{\|}}-1=N_{\|} \delta_{\mathbf{q}_{\|, 0}}-1,
$$

with $N_{\|}$denoting the total number of sites in a layer, Eq. (A2) can be expressed as

$$
S(\mathbf{q})=\frac{W^{2}}{12}\left[\theta+(1-\theta)^{2} N_{\|} \delta_{\mathbf{q}_{\|}, 0}\right] .
$$

In SCBA, the self-energy is therefore

$$
\begin{aligned}
\Sigma_{\mathrm{p}} & =\sum_{\mathrm{q}}\langle v(\mathbf{q}) v(-\mathbf{q})\rangle_{c} R_{\mathrm{p}+\mathrm{q}} \\
& =\frac{1}{N} \sum_{\mathrm{q}} S(\mathbf{q}) R_{\mathrm{p}+\mathrm{q}} \\
& =\frac{W^{2} \theta^{2}}{12 N} \sum_{\mathbf{q}} R_{\mathrm{p}+\mathrm{q}}+\frac{W^{2}(1-\theta)^{2}}{12 N_{\perp}} \sum_{\mathrm{q}_{\perp}} R_{\mathrm{p}+\mathrm{q}_{\perp}} .
\end{aligned}
$$

With the use of Eq. (7), it is easy to see that the first and second terms on the right-hand side of Eq. (A5) do not depend on $\mathbf{p}$ and $\mathbf{p}_{1}$, respectively. Thus the selfconsistent solution of Eq. (A5) leads to a self-energy that does not depend on $\mathbf{p}_{1}$, and Eq. (A5) can therefore be rewritten in the form of Eq. (8).

\section{APPENDIX B}

Here the expression $\phi_{x x}^{R A(0)}\left(0^{+}, E\right)$ of Eq. (12), due to the contribution from all ladder diagrams that contain only 1D scatterings (dotted vertex lines), is evaluated. The contributions from Figs. 1(a), 1(b), 1(c), etc., can be written down and summed as follows: 


$$
\begin{aligned}
& \delta_{x x}^{R A(0)}\left(0^{+}, E\right)=\frac{1}{4 \pi^{2}} \sum_{\mathbf{p}_{1} \mathrm{p}_{2}} v_{x}\left(\mathbf{p}_{1}\right) v_{x}\left(\mathbf{p}_{2}\right) R_{\mathrm{p}_{1}}^{+}(E) R_{\mathbf{p}_{1}}^{-}(E) \\
& \times\left[\delta_{\mathrm{p}_{1}, \mathrm{p}_{2}}+\frac{W^{2}(1-\theta)^{2} N_{\|}}{12 N}\left[\delta_{\left(\mathrm{p}_{1}\right)_{\|},\left(p_{2}\right)_{\|}} R_{\mathrm{p}_{2}}^{+}(E) R_{\mathrm{p}_{2}}^{-}(E)+\frac{W^{2}(1-\theta)^{2} N_{\|}}{12 N} \sum_{\mathrm{p}_{3}} \delta_{\left(\mathbf{p}_{1}\right)_{\|},\left(\mathrm{p}_{3}\right)_{\|}} R_{\mathrm{p}_{3}}^{+}(E)\right.\right. \\
& \left.\left.\times R_{\mathrm{p}_{3}}^{-}(E) \delta_{\left(\mathrm{p}_{3}\right)_{\|},\left(\mathrm{p}_{2}\right) \|} R_{\mathrm{p}_{2}}^{+}(E) R_{\mathrm{p}_{2}}^{-}(E) \cdots\right)\right] \\
& =\frac{1}{4 \pi^{2}} \sum_{\mathbf{p}_{1}, \mathbf{p}_{2}} v_{x}\left(\mathbf{p}_{1}\right) v_{x}\left(\mathbf{p}_{2}\right) R_{\mathbf{p}_{1}}^{+} R_{\mathbf{p}_{1}}^{-} \int \delta_{\mathrm{p}_{1}, \mathbf{p}_{2}}+\frac{W^{2}(1-\theta)^{2}}{12 N_{1}} \delta_{\left(\mathbf{p}_{1}\right)_{\|},\left(\mathrm{p}_{2}\right)_{\|}} R_{\mathrm{p}_{2}}^{+} R_{\mathrm{p}_{2}}^{-} \\
& \left.\times \frac{1}{1-\left[W^{2}(1-\theta)^{2} / 12 N_{1}\right] \sum_{\left(\mathrm{p}_{3}\right)_{1}} R_{\left(\mathrm{p}_{2}\right)_{\|}+\left(\mathrm{p}_{3}\right)_{1}}^{+} R_{\left(\mathrm{p}_{2}\right)_{\|}+\left(\mathrm{p}_{3}\right)_{\perp}}^{-}}\right) \\
& =\frac{1}{4 \pi^{2}} \sum_{\mathbf{p}_{1}} v_{x}^{2}\left(\mathbf{p}_{1}\right) R_{\mathbf{p}_{1}}^{+} p_{\mathbf{p}_{1}}^{-} \frac{1}{1-\left[W^{2}(1-\theta)^{2} / 12 N_{\perp}\right] \sum_{\left(\mathbf{p}_{3}\right)_{\perp}} R_{\left(\mathbf{p}_{1}\right)_{\|}+\left(p_{3}\right)_{\perp}}^{+} R_{\left(\mathbf{p}_{1}\right)_{\|}+\left(\mathbf{p}_{3}\right)_{\perp}}^{-}} \\
& =\frac{1}{4 \pi^{2}} \sum_{\mathbf{p}_{1}} v_{x}^{2}\left(\mathbf{p}_{1}\right) \frac{\Delta R_{\mathbf{p}_{1}}}{\Delta \Sigma_{\left(\mathbf{p}_{1}\right)_{\|}}} \frac{1}{1-\left[W^{2}(1-\theta)^{2} / 12 N_{\perp}\right] \sum_{\left(\mathbf{p}_{3}\right)_{\perp}}\left[\Delta R_{\left(\mathbf{p}_{1}\right)_{\|}+\left(\mathbf{p}_{3}\right)_{\perp}} / \Delta \Sigma_{\left(\mathbf{p}_{1}\right)_{\|}}\right]} \text {. }
\end{aligned}
$$

Here Eqs. (14) - (16) have been used in the last term of Eq. (B1). However, SCBA of Eq. (8) gives the relation

$$
\Delta \Sigma_{\mathbf{p}_{\|}}=\frac{W^{2} \theta^{2}}{12 N} \sum_{\mathrm{p}} \Delta R_{\mathrm{p}}+\frac{W^{2}(1-\theta)^{2}}{12 N_{\perp}} \sum_{(\mathrm{p})_{\perp}} \Delta R_{(\mathrm{p})_{\|}+(\mathrm{p})_{\perp}} \text {. }
$$

Substituting Eq. (B2) into Eq. (B1), yields

$$
\phi_{x x}^{R A(0)}\left(0^{+}, E\right)=\frac{3 N}{\pi^{2} W^{2} \theta^{2}} \frac{\sum_{\mathrm{p}_{1}} v_{x}^{2}\left(\mathrm{p}_{1}\right) \Delta R_{\mathrm{p}_{1}}(E)}{\sum_{\mathrm{p}} \Delta R_{\mathrm{p}}(E)} .
$$

Similarly,

$$
\begin{aligned}
\phi_{y y}^{R A(0)}\left(0^{+}, E\right) & =\phi_{y y}^{A R(0)}\left(0^{+}, E\right) \\
& =\phi_{x x}^{A R(0)}\left(0^{+}, E\right) \\
& =\phi_{x x}^{R A(0)}\left(0^{+}, E\right) .
\end{aligned}
$$

Equation (19) is obtained by substituting Eqs. (B3) and (B4) into Eq. (11).

\section{APPENDIX C}

To evaluate the maximally crossed diagrams shown in Fig. 2(b), the diagrams in the particle-hole channel is redrawn so that it becomes the particle-particle channel. The momentum of the lower lines are then reversed. By using the time-reversal symmetry, the resulting ladder diagrams become equivalent to the standard form and therefore can be summed. Following the above procedure, the diagram shown in Fig. 2(c) becomes the one shown in Fig. 2(h) where the momentum $\mathbf{p}_{3 \pm}$ are given by $\mathbf{p}_{3 \pm}=\mathbf{p}_{3 \pm} \mathbf{k} / 2$ with $\mathbf{k}=\mathbf{p}_{1}+\mathbf{p}_{2}$. Since the momentum is conserved at each vertex, i.e., $\mathbf{p}_{1}+\mathbf{p}_{3-}=\mathbf{p}_{3+}-\mathbf{p}_{2}$, it is easy to see from Appendix $A$ that this diagram gives a term

$$
\sum_{\mathrm{p}_{3}} S\left(\mathrm{p}_{1}-\mathrm{p}_{3+}\right) R_{\mathrm{p}_{3+}}^{+} R_{\mathrm{p}_{3-}}^{-} S\left(\mathrm{p}_{3+}-\mathrm{p}_{2}\right),
$$

where $S(\mathrm{q})$ is given by Eq. (A4). Similarly, the diagram in Fig. 2(d) can be converted to that of Fig. 2(i), giving

$$
\begin{aligned}
\sum_{\mathrm{p}_{3}, \mathrm{p}_{4}} S\left(\mathrm{p}_{1}-\mathrm{p}_{3+}\right) & R_{\mathrm{p}_{3+}}^{+} R_{\mathrm{p}_{3-}}^{-} \\
& \times S\left(\mathrm{p}_{3}-\mathrm{p}_{4}\right) R_{\mathrm{p}_{4+}}^{+} R_{\mathrm{p}_{4-}}^{-} S\left(\mathrm{p}_{4+}-\mathrm{p}_{2}\right) .
\end{aligned}
$$

By summing these diagrams to infinite order, the function $U^{(M)}$ can be written as

$$
\begin{aligned}
U^{(M)}\left(\mathbf{p}_{1}, \mathbf{p}_{2} ; \omega, E\right)= & \sum_{\mathbf{p}_{3}, \mathbf{p}_{4}} S\left(\mathbf{p}_{1}-\mathbf{p}_{3+}\right) \\
& \times \phi_{\mathbf{p}_{3}, p_{4}}^{(0)}(\mathbf{k} ; \omega, E) S\left(\mathbf{p}_{4+}-\mathbf{p}_{2}\right) \\
= & \sum_{\mathbf{p}_{3}, \mathbf{p}_{4}} S\left(\mathbf{p}_{3}+\mathbf{Q}\right) \phi_{\mathbf{p}_{3}, \mathbf{p}_{4}}^{(0)} S\left(\mathbf{Q}-\mathbf{p}_{4}\right)
\end{aligned}
$$

with

$$
\mathbf{Q}=\left(\mathbf{p}_{2}-\mathbf{p}_{1}\right) / 2,
$$

where the function $\phi_{\mathbf{p}_{3}, \mathrm{p}_{4}}^{(0)}(\mathbf{k} ; \omega, E)$ satisfies the following self-consistent equation

$$
\phi_{\mathrm{p}_{3}, \mathrm{p}_{4}}^{(0)}(\mathbf{k} ; \omega, E)=R_{\mathrm{p}_{3}+}^{+} R_{\mathrm{p}_{3-}}^{-}\left(\delta_{\mathrm{p}_{3}, \mathrm{p}_{4}}+\sum_{\mathrm{p}_{5}} S\left(\mathbf{p}_{3}-\mathbf{p}_{5}\right) \phi_{\mathbf{p}_{5}, \mathrm{p}_{4}}^{(0)}(\mathbf{k} ; \omega, E)\right)
$$


Substituting Eq. (A4) for the function $S$ into EQ. (C3) and (C5), we have, respectively,

$$
\begin{aligned}
U_{\left(\mathbf{p}_{1}, \mathbf{p}_{2} ; \omega, E\right)}^{(M)}=\left[\frac{W^{2}}{12 N}\right)^{2}\left(\theta^{4} \phi^{(0)}(\mathbf{k}, \omega, E)+\theta^{2}(1-\theta)^{2} N_{\|} \boldsymbol{\Sigma}_{\left(\mathbf{p}_{3}\right)_{1}}\left[\phi_{\left(\mathbf{p}_{3}\right)_{\perp}-\mathbf{Q}_{\|}}^{(0)}(\mathbf{k}, \omega, E)+\phi_{\left(\mathbf{p}_{3}\right)_{\perp}}^{(0)}+\mathbf{Q}_{\|}(\mathbf{k}, \omega, E)\right]\right. \\
\left.+(1-\theta)^{4} N_{\|}^{2} \sum_{\left.\left(\mathbf{p}_{3}\right)_{1}, p_{4}\right)_{\perp}} \phi_{\left(\mathbf{p}_{3}\right)_{\perp}-\mathbf{Q}_{\|},\left(\mathbf{p}_{4}\right)_{\perp}+\mathbf{Q}_{\|}}^{(0)}(\mathbf{k}, \omega, E)\right),
\end{aligned}
$$

and

$$
\phi_{\mathbf{p}_{3}, \mathbf{p}_{4}}^{(0)}(\mathbf{k} ; \omega, E)=R_{\mathbf{p}_{3+}}^{+} R_{\mathbf{p}_{3-}}^{-}\left(\delta_{\mathbf{p}_{3}, \mathbf{p}_{4}}+\frac{W^{2} \theta^{2}}{12 N} \phi_{\mathbf{p}_{4}}^{(0)}(\mathbf{k} ; \omega, E)+\frac{W^{2}(1-\theta)^{2}}{12 N_{\perp}} \sum_{\left(\mathbf{p}_{5}\right)_{\perp}} \phi_{\left(\mathbf{p}_{3}\right)_{\|}+\left(\mathbf{p}_{5}\right)_{\|}, \mathbf{p}_{4}}^{(0)}(\mathbf{k}, \omega, E)\right),
$$

where we have defined

$$
\phi^{(0)}(\mathbf{k} ; \omega, E)=\sum_{\mathbf{p}_{3}, \mathbf{p}_{4}} \phi_{\mathbf{p}_{3}, \mathbf{p}_{4}}^{(0)}(\mathbf{k}, \omega, E)
$$

and

$$
\phi_{\mathbf{p}_{3}}^{(0)}(\mathbf{k}, \omega, E)=\sum_{\mathbf{p}_{4}} \phi_{\mathbf{p}_{3}, \mathbf{p}_{4}}^{(0)}(\mathbf{k} ; \omega, E)=\sum_{\mathbf{p}_{4}} \phi_{\mathbf{p}_{4}, \mathbf{p}_{3}}^{(0)}(\mathbf{k}, \omega, E) .
$$

The symmetry of the function $\phi_{\mathbf{p}_{3}, \mathbf{p}_{4}}^{(0)}$ with respect to $\mathbf{p}_{3}$ and $\mathbf{p}_{4}$ can be seen by iterating Eq. (C5). Summing both sides of Eq. (C7) with respect to $\mathbf{p}_{4},\left(\mathbf{p}_{3}\right)_{1}, \mathbf{p}_{4}$, and $\mathbf{p}_{3}$, we obtain, respectively,

$$
\sum_{\left(\mathbf{p}_{3}\right)_{\perp}} \phi_{\mathbf{p}_{3}}^{(0)}(\mathbf{k} ; \omega, E)=\left(1+\frac{W^{2} \theta^{2}}{12 N} \phi^{(0)}(\mathbf{k} ; \omega, E)+\frac{W^{2}(1-\theta)^{2}}{12 N_{\perp}} \sum_{\left(\mathbf{p}_{3}\right)_{\perp}} \phi_{\mathbf{p}_{3+}}^{(0)}(\mathbf{k} ; \omega, E)\right) \sum_{\left(\mathbf{p}_{3}\right)_{\perp}} R_{\mathbf{p}_{3+}}^{+}\left(E_{+}\right) R_{\mathbf{p}_{3-}}^{-}\left(E_{-}\right),
$$

and

$$
\begin{aligned}
& \phi^{(0)}(\mathbf{k}, \omega, E)=\left(1+\frac{W^{2} \theta^{2}}{12 N} \phi^{(0)}(\mathbf{k} ; \omega, E)\right) \sum_{\mathbf{p}_{3}} R_{\mathrm{p}_{3+}}^{+}\left(E_{+}\right) R_{\mathbf{p}_{3-}}^{-}\left(E_{-}\right)+\frac{W^{2}(1-\theta)^{2}}{12 N_{\perp}} \\
& \times \sum_{\mathrm{p}_{3}} R_{\mathbf{p}_{3+}}^{+}\left(E_{+}\right) R_{\mathbf{p}_{3-}}^{-}\left(E_{-}\right) \sum_{\left(\mathbf{p}_{5}\right)_{\perp}} \phi_{\left.\left(\mathbf{p}_{3}\right)_{1}\right)}^{(0)}+\left(\mathbf{p}_{5}\right)_{\perp}(\mathbf{k}, \omega, E) .
\end{aligned}
$$

Solving Eq. (C10) for $\Sigma_{\left(\mathrm{p}_{3}\right)_{1}} \phi_{\mathrm{p}_{3}}^{(0)}$ and then substituting it into Eq. (C11) yields

$$
\sum_{\left(\mathrm{p}_{3}\right)_{1}} \phi_{\left(\mathrm{p}_{3}\right)_{\|}+\left(\mathbf{p}_{3}\right)_{\perp}}^{(0)}(\mathbf{k}, \omega, E)=\frac{\sum_{\left(\mathbf{p}_{3}\right)_{\perp}} R_{\mathbf{p}_{3+}}^{+} R_{\mathrm{p}_{3-}}^{-}\left[1+\left(W^{2} \theta^{2} / 12 N\right) \phi^{(0)}\right]}{1-\left[W^{2}(1-\theta)^{2} / 12 N_{\perp}\right] \sum_{\mathrm{p}_{3}} R_{\mathrm{p}_{3+}}^{+} R_{\mathrm{p}_{3-}}^{-}}
$$

and

$$
\phi^{(0)}(\mathbf{k}, \omega, E)=\frac{\sum_{\mathrm{p}_{3}} R_{\mathrm{p}_{3+}}^{+} R_{\mathrm{p}_{3-}-}^{-} \frac{1}{1-\left[W^{2}(1-\theta)^{2} / 12 N_{\perp}\right] \sum_{\left(\mathrm{p}_{4}\right)_{1}} R_{\left[\left(\mathrm{p}_{3}\right)_{\|}+\left(\mathrm{p}_{4}\right)_{1}\right]_{+}}^{+} R_{\left[\left(\mathrm{p}_{3}\right)_{\|}+\left(\mathrm{p}_{4}\right)_{1}\right]_{-}}}}{1-\left(W^{2} \theta^{2} / 12 N\right) \sum_{\mathrm{p}_{3}} R_{\mathrm{p}_{3+}}^{+} R_{\mathrm{p}_{3}-}^{-} \frac{1}{1-\left[W^{2}(1-\theta)^{2} / 12 N_{\perp}\right] \sum_{\left(\mathrm{p}_{4}\right)_{1}} R_{\left[\left(\mathrm{p}_{3}\right)_{\|}+\left(\mathrm{p}_{4}\right)_{1}\right]_{+}}^{+} R_{\left[\left(\mathrm{p}_{3}\right)_{\|}+\left(\mathrm{p}_{4}\right)_{1}\right]_{-}}^{-}} .} .
$$

The functions $\phi_{\left(\mathbf{p}_{3}\right)_{1} \pm \mathbf{Q}_{\|}}^{(0)}$ in Eq. (C6) are obtained by setting $\left(\mathbf{p}_{3}\right)_{\|}= \pm \mathbf{Q}_{\|}$in Eq. (C12). With the use of the relations $\mathbf{p}_{3} \pm=\mathbf{p}_{3} \pm \mathbf{k} / 2=p_{3} \pm\left(\mathbf{p}_{1}+\mathbf{p}_{2}\right) / 2$ and Eq. (C4), we find

$$
\sum_{\left(\mathbf{p}_{3}\right)_{\perp}} \phi_{\left(\mathbf{p}_{3}\right)_{1}-\mathbf{Q}_{\|}}^{(0)}(\mathbf{k} ; \omega, E)=F\left(\left(\mathbf{p}_{1}\right)_{\|},\left(\mathbf{p}_{2}\right)_{\|}, \mathbf{k}_{1} ; \omega, E\right)\left(1+\frac{W^{2} \theta^{2}}{12 N} \phi^{(0)}(\mathbf{k} ; \omega, E)\right),
$$

and

$$
\sum_{\left(\mathbf{p}_{3}\right)_{\perp}} \phi_{\left(\mathbf{p}_{3}\right)_{1}+\mathbf{Q}_{\|}}^{(0)}(\mathbf{k} ; \omega, E)=F\left(\left(\mathbf{p}_{2}\right)_{\|},\left(\mathbf{p}_{1}\right)_{\|}, \mathbf{k}_{1} ; \omega, E\right)\left(1+\frac{W^{2} \theta^{2}}{12 N} \phi^{(0)}(\mathbf{k}, \omega, E)\right),
$$


with

$$
F\left(\left(\mathbf{p}_{1}\right)_{\|},\left(\mathbf{p}_{2}\right)_{\|}, \mathbf{k}_{1} ; \omega, E\right)=\frac{\sum_{\left(\mathbf{p}_{3}\right)_{\perp}} R_{\left(\mathbf{p}_{1}\right)_{\|}+\left(\mathbf{p}_{3}\right)_{\perp}+\mathbf{k}_{1} / 2}^{+}\left(E_{+}\right) R_{-\left(\mathbf{p}_{2}\right)_{\|}+\left(\mathbf{p}_{3}\right)_{\|}-\mathbf{k}_{\perp} / 2}^{-}\left(E_{-}\right)}{1-\left[W^{2}(1-\theta)^{2} / 12 N_{\perp}\right] \sum_{\left(\mathbf{p}_{3}\right)_{\perp}} R_{\left(\mathbf{p}_{1}\right)_{\|}^{+}+\left(\mathbf{p}_{3}\right)_{\perp}+\mathbf{k}_{\perp} / 2}^{+}\left(E_{+}\right) R_{-\left(\mathbf{p}_{2}\right)_{\|}+\left(\mathbf{p}_{3}\right)_{\perp}-\mathbf{k}_{\perp} / 2}^{-}\left(E_{-}\right)} .
$$

Similarly, the last term in Eq. (C6) can be obtained by setting $\left(\mathbf{p}_{3}\right)_{\|}=-\mathbf{Q}_{\|},\left(\mathbf{p}_{4}\right)_{\|}=\mathbf{Q}_{\|}$in Eq. (C7) and summing both sides with respect to $\left(\mathbf{p}_{3}\right)_{1}$ and $\left(\mathbf{p}_{4}\right)_{\perp}$. We find

$$
\sum_{\left(\mathbf{p}_{3}\right)_{1},\left(\mathbf{p}_{4}\right)_{\perp}} \phi_{\left(\mathbf{p}_{3}\right)_{1}-\mathbf{Q}_{\|},\left(\mathbf{p}_{4}\right)_{\perp}+\mathbf{Q}_{\|}}^{(0)}(\mathbf{k}, \omega, E)=F\left(\left(\mathbf{p}_{1}\right)_{\|},\left(\mathbf{p}_{2}\right)_{\|}, \mathbf{k} ; \omega, E\right)\left(\delta_{\left(\mathbf{p}_{1}\right)_{\|},\left(\mathbf{p}_{2}\right)_{\|}}+\frac{W^{2} \theta^{2}}{12 N} \sum_{\left(\mathbf{p}_{4}\right)_{\perp}} \phi_{Q_{\|}}^{(0)}+\left(\mathbf{p}_{4}\right)_{\perp}(\mathbf{k}, \omega, E)\right) .
$$

Substituting Eqs. (C14), (C15), and (C17) into Eq. (C6), we obtained

$$
\begin{aligned}
U^{(M)}\left(\mathbf{p}_{1}, \mathbf{p}_{2} ; \omega, E\right)=\left[\frac{W^{2}}{12 N}\right]^{2}[ & {\left[\theta^{2}+(1-\theta)^{2} \theta^{2} \frac{W^{2}}{12 N_{\perp}} F\left(\left(\mathbf{p}_{1}\right)_{\|},\left(\mathbf{p}_{2}\right)_{\|}, \mathbf{k}_{\perp} ; \omega, E\right)\right] } \\
& \times\left[\theta^{2}+(1-\theta)^{2} \theta^{2} \frac{W^{2}}{12 N} F\left(\left(\mathbf{p}_{2}\right)_{\|},\left(\mathbf{p}_{1}\right)_{\|}, \mathbf{k}_{\perp} ; \omega, E\right)\right] \phi^{(0)}(\mathbf{k} ; \omega, E) \\
& +(1-\theta)^{4} N_{\|}^{2} F\left(\left(\mathbf{p}_{1}\right)_{\|},\left(\mathbf{p}_{2}\right)_{\|}, \mathbf{k}_{\perp} ; \omega, E\right) \delta_{\left(\mathbf{p}_{1}\right)_{\|},\left(\mathbf{p}_{2}\right)_{\|}} \\
& +\theta^{2}(1-\theta)^{2} N_{\|}\left[F\left(\left(\mathbf{p}_{1}\right)_{\|},\left(\mathbf{p}_{2}\right)_{\|}, \mathbf{k}_{\perp} ; \omega, E\right)\right. \\
& \left.+F\left(\left(\mathbf{p}_{2}\right)_{\|},\left(\mathbf{p}_{1}\right)_{\|}, \mathbf{k}_{1} ; \omega, E\right)\right]+\theta^{2}(1-\theta)^{4} \frac{W^{2} N_{\|}^{2}}{12 N} F\left(\left(\mathbf{p}_{1}\right)_{\|},\left(\mathbf{p}_{2}\right)_{\|}, \mathbf{k}_{\perp} ; \omega, E\right) \\
& \left.\times F\left(\left(\mathbf{p}_{2}\right)_{\|},\left(\mathbf{p}_{1}\right)_{\|}, k_{\perp} ; \omega, E\right)\right] .
\end{aligned}
$$

The first term of Eq. $(\mathrm{C} 18)$ contains a factor $\phi^{(0)}(\mathbf{k} ; \omega, E)$, which is shown to be a 3D diffusion pole in Appendix D. The second term is noted to contain a factor $(1-\theta)^{4}$ coming from the 1D scattering alone. The last two terms, which vanish in both the $\theta \rightarrow 0$ and $\theta \rightarrow 1$ limits, arise from a mixture of 3D and 1D scattering. Without losing the qualitative feature of the problem, these two terms have been ignored in Eq. (26) of the main text.

\section{APPENDIX D}

In this Appendix, we will show that in the hydrodynamic limit the functions $\phi^{(0)}$ of Eq. (28) and $F$ of Eq. (31) can be written in the forms of Eqs. (32) and (33), respectively. Using Taylor's expansion up to first order in $\omega$ and second order in $k$, we have

$$
\begin{aligned}
R_{\mathrm{p}_{+}}^{+}\left(E_{+}\right)=R_{\mathrm{p}+\mathrm{k} / 2}(E+\omega / 2) & =\frac{1}{E+\omega / 2-\varepsilon(\mathbf{p}+\mathbf{k} / 2)-\Sigma_{\mathrm{p}+\mathrm{k} / 2}^{+}(E+\omega / 2)} \\
& \cong R_{\mathrm{p}}^{+}(E)+\frac{\partial R_{\mathrm{p}}^{+}(E)}{\partial E} \frac{\omega}{2}+\nabla_{\mathrm{p}} R_{\mathrm{p}}^{+}(E) \cdot \frac{\mathbf{k}}{2}+\frac{1}{2} \sum_{j, l=x, y, z} \frac{\partial}{\partial p_{j}} \frac{\partial}{\partial p_{l}} R_{\mathrm{p}}^{+}(E) \frac{k_{J}}{2} \frac{k_{l}}{2} .
\end{aligned}
$$

The function $R_{\mathrm{p}^{-}}^{-}(E)$ can also be expanded in a similar way. By using these expansions, the function $A$ of Eq. (30) becomes

$$
\begin{aligned}
A\left(\left(\mathbf{p}_{3}\right)_{\|} ; \omega, E\right) \cong \frac{W^{2}(1-\theta)^{2}}{12 N_{\perp}} \sum_{\left(\mathbf{p}_{4}\right)_{\perp}}[ & \frac{\Delta R_{\mathrm{q}}}{\Delta \Sigma_{\left(\mathbf{p}_{3}\right)_{\|}}}-\frac{\left(\Delta R_{\mathrm{q}}\right)^{2}}{\Delta \Sigma_{\left(\mathbf{p}_{3}\right)_{\|}}} \frac{\omega}{2}-\frac{1}{2}\left[\frac{\Delta R_{\mathrm{q}}}{\Delta \Sigma_{\left(\mathbf{p}_{3}\right)_{\|}}}\right)^{2} v_{\perp}^{2}\left(\left(\mathbf{p}_{4}\right)_{\perp}\right) k_{\perp}^{2}+\frac{\left(\Delta R_{\mathrm{q}}\right)^{2}}{\Delta \Sigma_{\left(\mathbf{p}_{3}\right)_{\|}}} \boldsymbol{v}_{\|}\left(\left(\mathbf{p}_{3}\right)_{\|}\right) \cdot \frac{\mathbf{k}_{\|}}{2} \\
& +\frac{1}{8} \sum_{j, l=x, y}\left[R_{\mathrm{q}}^{+} \frac{\partial}{\partial p_{j}} \frac{\partial}{\partial p_{l}} R_{\mathrm{q}}^{-} k_{j} k_{l}+R_{\mathrm{q}}^{-} \frac{\partial}{\partial p_{j}} \frac{\partial}{\partial p_{l}} R_{\mathrm{q}}^{+} k_{j} k_{l}\right) \\
& \left.-\frac{1}{4} \sum_{i, l_{1}=x, y} \frac{\partial}{\partial p_{j}} R_{\mathrm{q}}^{+} \frac{\partial}{\partial p_{l}} R_{\mathrm{q}}^{-} k_{j} k_{l}\right],
\end{aligned}
$$

where we have defined $\mathbf{q}$ as $\left(\mathbf{p}_{3}\right)_{\|}+\left(\mathbf{p}_{4}\right)_{1}$, and Eqs. (14) and (15) have been used in deriving Eq. (D2). The terms linear in $k_{Z}$ disappear under summation of $\left(\mathbf{p}_{4}\right)_{1}$ due to the accompanying factor $v\left(\left(\mathbf{p}_{4}\right)_{1}\right)$. Since the first term of $U^{(M)}$ [in Eq. (26)] is basically due to $3 \mathrm{D}$ isotropic scattering, we can expand the function $(1-\boldsymbol{A})^{-1}$ in Eq. (28) in powers of $(1-\theta)^{2}$. 
With the use of Eq. (8), we find from Eq. (D2), with some manipulation,

$$
\begin{aligned}
{\left[1-A\left(\left(\mathbf{p}_{3}\right)_{\|} ; \omega, E\right)\right]^{-1} \cong } & \frac{\Delta \Sigma_{\left(\mathbf{p}_{3}\right)_{\|}}}{M}-\left(\frac{\Delta \Sigma_{\left(\mathbf{p}_{3}\right)_{\|}}}{M}\right)^{2} \frac{W^{2}(1-\theta)^{2}}{12 N_{\perp}} \\
& \times \sum_{\left(\mathbf{p}_{4}\right)_{\perp}}\left[\frac{\left(\Delta R_{\mathbf{q}}\right)^{2}}{\Delta \Sigma_{\left(\mathbf{p}_{3}\right)_{\|}}} \frac{\omega}{2}+\frac{1}{2}\left[\frac{\Delta R_{\mathbf{q}}}{\Delta \Sigma_{\left(\mathbf{p}_{3}\right)_{\|}}}\right]^{2} v_{\perp}^{2}\left(\left(\mathbf{p}_{4}\right)_{\perp}\right) k_{\perp}^{2}-\frac{\left(\Delta R_{\mathbf{q}}\right)^{2}}{\Delta \Sigma\left(\mathbf{p}_{3}\right)_{\|}} \mathbf{v}_{\|}\left(\left(\mathbf{p}_{3}\right)_{\|}\right) \cdot \frac{\mathbf{k}_{\|}}{2}\right] \\
& -\frac{1}{8} \sum_{j, l=x, y}\left[R_{\mathrm{q}}^{+} \frac{\partial}{\partial p_{j}} \frac{\partial}{\partial p_{l}} R_{\mathbf{q}}^{-} k_{j} k_{l}+R_{\mathbf{q}}^{-} \frac{\partial}{\partial p_{j}} \frac{\partial}{\partial p_{l}} R_{\mathrm{q}}^{+} k_{j} k_{l}\right) \\
& +\frac{1}{4} \sum_{i, l=x, y} \frac{\partial}{\partial p_{j}} R_{\mathbf{q}}^{+} \frac{\partial}{\partial p_{l}} R_{\mathbf{q}}^{-}+\frac{\Delta \Sigma_{\left(\mathbf{p}_{3}\right)_{\|}}}{8 M^{3}}\left[\frac{W^{2}(1-\theta)^{2}}{12 N_{\perp}} \sum_{\left(\mathbf{p}_{4}\right)_{\perp}}\left(\Delta R_{\mathbf{q}}\right)^{2}\right]^{2} v_{\|}^{2}\left(\left(\mathbf{p}_{3}\right)_{\|}\right) k_{\|}^{2},
\end{aligned}
$$

with

$$
M=\left(\frac{-\pi i}{6}\right) W^{2} \theta^{2} \rho(E) .
$$

Similarly, the function $R_{\mathrm{p}_{3+}}^{+}\left(E_{+}\right) R_{\mathrm{p}_{3-}^{-}}^{-}\left(E_{-}\right)$is expanded to the order $\omega$ and $k^{2}$. With the use of the Eq. (40), after some lengthy algebraic manipulations, the following result is obtained

$$
\begin{aligned}
\frac{W^{2} \theta^{2}}{12 N} \sum_{\mathrm{p}_{3}} R_{\mathrm{p}_{3+}}^{+}\left(E_{+}\right) R_{\mathrm{p}_{3-}}^{-}\left(E_{-}\right)\left[1-A\left(\left(\mathbf{p}_{3}\right)_{\|} ; \omega, E\right)\right]^{-1} \cong 1-\frac{6}{\pi W^{2} \theta^{2} \rho(E)} & -i \omega+\frac{1}{2 \pi \rho N} \sum_{\mathrm{p}_{3}} v_{z}^{2}\left(\left(\mathbf{p}_{3}\right)_{1}\right) \frac{\Delta R_{\mathrm{p}_{3}}}{\Delta \Sigma_{\left(\mathrm{p}_{3}\right)}} k_{\perp}^{2} \\
& \left.+\frac{3 v_{\|}^{2}(E) k_{\|}^{2}}{\pi W^{2} \theta^{2} \rho(E)}\right) .
\end{aligned}
$$

By substituting Eq. (D5) into Eq. (28), the leading term yields Eq. (32). ly

The expansion of the function $F$ in Eq. (31) is much simpler due to the absence of $\mathbf{k}_{\|}$. Equation (D1) gives immediate-

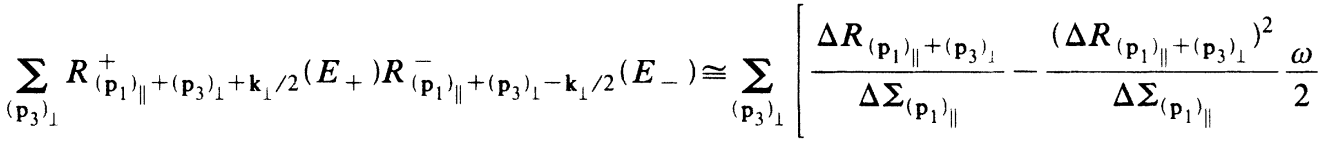

$$
\begin{aligned}
& \left.-\frac{1}{2}\left[\frac{\Delta \boldsymbol{R}_{\left(\mathrm{p}_{1}\right)_{\|}+\left(\mathrm{p}_{3}\right)_{1}}}{\Delta \Sigma_{\left(\mathrm{p}_{1}\right)_{\|}}}\right]^{2} v_{1}^{2}\left(\left(p_{3}\right)_{\perp}\right) k_{\perp}^{2}\right] \text {. }
\end{aligned}
$$

Substituting Eq. (D6) into Eq. (31) and using Eq. (8), we obtain Eq. (33) as the leading term.

${ }^{1}$ See, for example, P. A. Lee and T. V. Ramakrishnan, Rev. Mod. Phys. 57, 287 (1985).

${ }^{2}$ E. Abrahams, P. W. Anderson, D. C. Licciardello, and T. V. Ramakrishnan, Phys. Rev. Lett. 42, 673 (1979).

${ }^{3}$ D. Vollardt and P. Wolfle, Phys. Rev. Lett. 48, 699 (1982).

${ }^{4}$ P. Lee and D. Fisher, Phys. Rev. Lett. 47, 882 (1981).

${ }^{5}$ A. MacKinnon and B. Kramer, Phys. Rev. Lett. 47, 1546 (1981); Z. Phys. B 53, 1 (1983).

${ }^{6}$ A. D. Zdetsis, C. H. Soukoulis, E. N. Economou, and G. S. Grest, Phys. Rev. B 32, 7811 (1985).

${ }^{7}$ P. W. Anderson, Phys. Rev. 109, 1492 (1958).

${ }^{8}$ See also Percolation, Localization, and Superconductivity, edited by A. M. Goldman and S. A. Wolf (Plenum, New York, 1984).

${ }^{9}$ W. Xue, P. Sheng, Q. J. Chu, and Z. Q. Zhang, Phys. Rev. Lett. 63, 2837 (1989).
${ }^{10}$ D. Vollhardt and P. Wolfle, Phys. Rev. Lett. 45, 842 (1980); Phys. Rev. B 22, 4666 (1980).

${ }^{11}$ E. N. Economou, C. M. Soukoulis, and A. D. Zdetsis, Phys. Rev. B 30, 1686 (1984).

${ }^{12}$ Q. J. Chu and Z. Q. Zhang, Phys. Rev. B 38, 4906 (1988).

${ }^{13}$ For literature on classical wave localization, see Scattering and Localization of Classical Waves in Random Media, edited by P. Sheng (World Scientific, Singapore, 1990).

${ }^{14}$ S. John, H. Sompolinsky, and M. J. Stephen, Phys. Rev. B 27, 5592 (1983).

${ }^{15}$ Q. J. Chu and Z. Q. Zhang, Phys. Rev. B 39, 7120 (1989).

${ }^{16}$ W. Apel and T. M. Rice, J. Phys. C 16, L1151 (1983).

${ }^{17}$ D. J. Bishop, R. C. Dynes, B. J. Lin, and D. C. Tsui, Phys. Rev. B 30, 3539 (1984).

${ }^{18}$ P. Wolfle and R. N. Bhatt, Phys. Rev. B 30, 3542 (1984).

${ }^{19}$ R. N. Bhatt, P. Wolfle, and T. V. Ramakrishnan, Phys. Rev. B 
32, 569 (1985).

${ }^{20}$ Q. Li, C. M. Soukoulis, E. N. Economou, and G. S. Grest, Phys. Rev. B 40, 2825 (1989).

${ }^{21}$ R. Kubo, J. Phys. Soc. Jpn. 12, 570 (1957).

${ }^{22}$ E. N. Economou, Green's Function in Quantum Physics, 2nd ed. (Springer-Verlag, New York, 1983), Chap. 7.

${ }^{23}$ I. Sadakata, J. Phys. Soc. Jpn. 54, 736 (1985).

${ }^{24}$ R. Bulka, B. Kramer, and A. MacKinnon, Z. Phys. B 60, 13 (1985).

${ }^{25}$ B. Bulka, M. Schreiber, and B. Kramer, Z. Phys. B 66, 21
(1987).

${ }^{26} \mathrm{P}$. Wolfle and D. Vollhardt, Anderson Localization, edited by Y. Nagaoka and H. Fukuyama (Springer-Verlag, Berlin, 1982).

${ }^{27}$ P. Sheng and Z. Q. Zhang, Phys. Rev. Lett. 57, 1879 (1986).

${ }^{28}$ P. Sheng, Z. Q. Zhang, B. White, and G. Papanicolaou, Phys. Rev. Lett. 57, 1000 (1986).

${ }^{29}$ B. White, P. Sheng, Z. Q. Zhang, and G. Papanicolaou, Phys. Rev. Lett. 59, 1918 (1987). 